\title{
1 A cross-species neural integration of gravity for motor optimisation
}

2

3 Jeremie Gaveau ${ }^{1,2}$, Sidney Grospretre ${ }^{1,3}$, Dora Angelaki ${ }^{4}$, Charalambos Papaxanthis ${ }^{1 *}$ 4

1. INSERM U1093-CAPS, Université Bourgogne Franche-Comté, UFR des Sciences du Sport, F-21000 Dijon, France

2. Baylor College of Medicine, Department of Neuroscience, Houston, TX, USA

3. EA4660-C3S Laboratory-Culture, Sport, Health and Society Univ. Bourgogne Franche-Comté, Besançon, France

4. New York University, New York City, NY 10003

11

$12 *$ These authors have equally contributed to the paper

13

14 Corresponding author: Jeremie Gaveau (jeremie.gaveau@u-bourgogne.fr) 


\section{Abstract}

16 Recent kinematic results, combined with model simulations, have provided support for the

17 hypothesis that the human brain uses an internal model of gravity to shape motor patterns that

18 minimise muscle effort. Because many different muscular activation patterns can give rise to

19 the same trajectory, here we analyse muscular activation patterns during single-degree-of-

20 freedom arm movements in various directions, which allow to specifically investigating

21 gravity-related movement properties. Using a well-known decomposition method of tonic and

22 phasic electromyographic activities, we demonstrate that phasic EMGs present systematic 23 negative phases. This negativity demonstrates that gravity effects are harvested to save 24 muscle effort and reveals that the brain implements an optimal motor plan using gravity to 25 accelerate downward and decelerate upward movements. Furthermore, for the first time, we 26 compare experimental findings in humans to monkeys, thereby generalising the Effortoptimization strategy across species. 


\section{Introduction}

The ability to purposely move one's own body is a critical survival function that humans and animals master with apparent ease. However, even the most straightforward body limb movement entails inherent difficulties for which the motor system has evolved sophisticated solutions (Franklin and Wolpert, 2011; Shadmehr and Wise, 2005; Shadmehr et al., 2010; Wolpert and Ghahramani, 2000). Amongst others, one of these solutions is to learn and store internal models to disambiguate sensory information and to predict forthcoming movement dynamics. On earth, a pervasive component affecting perception and motion is gravity (Bringoux et al., 2012; Dakin and Rosenberg, 2018; Elmore et al., 2014; Gaveau et al., 2011, 2016; Jorges and Lopez-Moliner, 2017; Klein et al., 2018; Lacquaniti et al., 2013; McIntyre et al., 2001; Van Pelt et al., 2005; Rosenberg and Angelaki, 2014; Saradjian et al., 2014; La Scaleia et al., 2019; Senot et al., 2005; Tajadura-Jimenez et al., 2018; De Vrijer et al., 2008). Studies in both humans and non-human primates have provided strong evidence that the brain evolved an internal representation of gravity. This representation is thought to involve neural computations of the brain stem, the cerebellum, the vestibular cortex, and the anterior thalamus (Angelaki et al., 2004; Indovina et al., 2005; Laurens et al., 2013a, 2016; Miller et al., 2008; Rousseau et al., 2016). Although the neural representation of gravity is well documented, how it may benefit the production of suitable motor commands is unclear. Yet, living organisms produce successful movements while facing gravity effects every day. It is of critical importance to shed light on the neural computations that underpin motor planning and control in the gravity field.

When moving our body limbs, the brain must generate neural commands that consider both inertial forces and gravity forces. Functional segregation of the inertial forces - related to the velocity and acceleration of the limb - and the gravity forces - related to the position of the limb - was long assumed (Atkeson and Hollerbach, 1985; Flanders and Herrmann, 1992; Hollerbach and Flash, 1982). According to this assumption, the internal model of gravity is used to compensate for the gravity force throughout the entire movement. That is, neural commands produce a muscular force that is equal and opposite to the gravity force. Such a neural policy is thought to facilitate the production of accurate movements to changing directions, amplitudes, durations, and loads, by merely scaling the inertial-dependent part of the motor command. Albeit based upon old literature, this influential Compensation hypothesis still guides current research in various fields such as motor control (d'Avella et al., 2008; Guigon et al., 2007; Kadmon Harpaz et al., 2014; Olesh et al., 2017; Russo et al., 
2014), movement perception (Cook et al., 2013; Edey et al., 2019) or neuro-rehabilitation (Krabben et al., 2012; Prange et al., 2009a, 2009b, 2012; Raj et al., 2019).

On the other hand, recent kinematic results challenged this prevalent theory by revealing velocity profiles for mono-articular arm movements whose temporal structure changes according to movement direction relative to the gravity vertical (Gaveau and Papaxanthis, 2011; Gaveau et al., 2014, 2016; Gentili et al., 2007; Le Seac'h and McIntyre, 2007). This finding is straightforward because, during mono-articular movements, only the gravity force changes with movement direction; inertial forces are direction-independent. Thus, this observation contradicts the fundamental premise of the Compensation hypothesis, which assumes direction-invariant kinematics. In contrast, the reported direction-dependent kinematics of human single-joint rotations are consistent with an optimal control strategy that discounts muscle effort (Berret et al., 2008; Crevecoeur et al., 2009; Gaveau et al., 2014, 2016). Such Effort-optimization hypothesis assumes an internal model of gravity to take advantage of its effects rather than to compensate for them.

During adaptation to microgravity, the properties of arm movements further supported this Effort-optimization hypothesis. As predicted by an optimisation model, the directiondependence of arm movements progressively vanished in microgravity (Gaveau et al., 2016). This contrasts to the traditional compensation view that the brain uses internal models of perturbing forces for their compensation, such that stereotypic trajectories can be maintained (Atkeson and Hollerbach, 1985; Hollerbach and Flash, 1982; Shadmehr and Mussa-Ivaldi, 1994). A more recent view is that motor adaptation constitutes a re-optimisation process whereby newly constructed/calibrated internal models generate newly shaped trajectories (Gaveau et al., 2016; Izawa et al., 2008; Selinger et al., 2015; Snaterse et al., 2011).

Support for the Effort-optimization hypothesis is so far limited to kinematic findings. This limitation is problematic because of the redundancy between the muscular level and the kinematic level (Bernstein, 1967). Because many different muscular activation patterns can give rise to the same trajectory, using kinematic data exclusively to infer central processes is insufficient (Burdet et al., 2001; Hagen and Valero-Cuevas, 2017). Establishing that direction-dependent kinematics truly reflects motor commands that discount muscle effort requires additional evidence from muscle dynamics. Here we analyse muscular activation patterns during single-degree-of-freedom arm movements performed in various directions. Furthermore, for the first time, we compared experimental findings in humans to monkeys, thereby generalising the Effort-optimization strategy across species. 


\section{Results}

We trained three rhesus monkeys to perform earth-vertical and earth-horizontal arm movements around the shoulder joint (Figure 1A). We also asked two groups of humans to perform earth-vertical and earth-horizontal arm movements from two different body orientations (seated upright and $90^{\circ}$ tilted in roll). Arm movements were parallel to the participants' head/feet body axis in a first group (ego-parallel group, n=8, Figure 1B) and perpendicular to it in a second group (ego-perpendicular group, $n=8$, Figure $1 \mathrm{C}$ ). Comparison between the ego-parallel group and the ego-perpendicular group allows dissociation of arm movement direction in body-centred and gravity-centred frames of reference.

Monkey and human arm movement kinematics follow similar direction-dependent asymmetries

Reaching arm movements typically exhibit bell-shaped velocity profiles (Kelso et al., 1979). Velocity first rises to a peak (acceleration phase) and then declines back to zero (deceleration phase, see Figure 2A-B). As previously reported, single-degree-of-freedom human arm movements show direction-dependent asymmetries in the earth-vertical but not the earth-horizontal plane (Gentili et al., 2007; Le Seac'h and McIntyre, 2007). A shorter and steeper acceleration profile for upward than for downward movements characterises these direction-dependent asymmetries (rise to peak velocity in Figure 2A-B, top traces). Because previous work already demonstrated that this effect was independent of body orientation, Figure 2 presents averaged results across both groups of humans (Fig 2, Suppl. Table 1 and Figure 2, Suppl. Table 2 provides results and statistics for each group). We found that monkeys also exhibit direction-dependent arm kinematics in the vertical plane only (Figure 2A-B, bottom traces). As in humans, the acceleration duration is shorter and steeper for upward than for downward movements.

This motor behaviour was quantified using multiple parameters (see Fig. 2, Suppl. Figure 1A and Fig. 2, Suppl. Table 1). The peak acceleration (which quantifies the steepness of the acceleration phase) and the duration to peak velocity (which specifies the length of the acceleration phase) illustrate this result in Figure 2C-F. Although movement duration and amplitude were similar for all directions ( $\mathrm{P}>0.19$ in all cases; see Fig. 2, Suppl. Table 2 for all statistical comparisons), a significant main effect of direction was observed for peak acceleration, peak velocity, relative time to peak acceleration, and relative time to peak velocity ( $\mathrm{P}<0.003$ in all cases). Post-hoc comparisons, between opposite directions, yielded 
significant effects in the earth-vertical but not the earth-horizontal plane $(\mathrm{P}<0.02$ in all cases). Thus, kinematic asymmetries in monkeys are identical to those previously known in humans.

Theoretical models minimising muscle effort predict these asymmetries (Berret et al., 2008; Crevecoeur et al., 2009; Gaveau et al., 2014, 2016). According to such models, the brain takes advantage of gravity effects to accelerate the arm downwards and decelerate the arm upwards. Next, we examine muscular activation patterns to understand the production of direction-dependent arm kinematics further. Because of the redundancy between the muscular level and the kinematic level (Bernstein, 1967), many different muscular activation patterns can give rise to the same velocity profile (Burdet et al., 2001; Hagen and Valero-Cuevas, 2017). Does the neural integration of gravity truly blossom into muscular activation patterns that discount muscle effort?

\section{Phasic EMG activity supports the Effort-optimization hypothesis}

Two types of muscles can contribute to the motion of the arm in the earth-vertical plane: those that pull against gravity (towards positive y-axis in Figure 1) and those that pull with gravity (towards negative y-axis). Hereafter, the first type is named antigravity muscles, and the second type is named gravity muscles. In the earth-horizontal plane, to produce the arm motion, the muscles pull perpendicularly to gravity. Hereafter, the muscles pulling towards the positive z-axis are named rightward-muscles, and the opposite ones are named leftward-muscles.

We applied a simple and widely-used decomposition method to isolate the tonic (gravity-dependent force) and the phasic (inertial-dependent force) EMG components from the full EMG signal (Buneo et al., 1994; d'Avella et al., 2006, 2008; Flanders and Herrmann, 1992; Flanders et al., 1994, 1996; Olesh et al., 2017; Prange et al., 2009b, 2012; Russo et al., 2014). The tonic component emanates from the motionless rest-periods before and after the movement (Fig. 2 Suppl. Figure 1 B, D). The phasic component results from the subtraction of the tonic activity from the total EMG (Fig. 2 Suppl. Figure 1 C-E). This subtraction stems from the Compensation hypothesis according to which the tonic would compensate for the gravity force, whereas the phasic would produce the change in velocity (Atkeson and Hollerbach, 1985; Flanders and Herrmann, 1992; Hollerbach and Flash, 1982). Here we take advantage of this method to test straightforward predictions of both the Compensation and the Effort-optimization hypotheses.

The straightforward prediction from the Compensation hypothesis is that phasic EMG activity of antigravity muscles is always positive when the arm is moving. That is, for 
162 antigravity muscles to compensate for the gravity force, the total activity in antigravity muscles should be at least equal to the tonic level over the entire movement amplitude. The straightforward prediction from the Effort-optimization hypothesis is that the phasic component of antigravity muscles exhibits periods of negativity. Inevitably, for gravity force to assist the arm motion, the antigravity muscle activity should drop below the tonic level that would be required to compensate it.

In accord with the Effort-optimization hypothesis, negative periods were frequent for antigravity muscles (Figure 3A, B; purple). The negativity of antigravity muscles precisely occurred when gravity effects could assist movement; that is, during the acceleration of downward movements (Figure 3A) and the deceleration of upward movements (Figure 3B). During those periods, the arm presumably falls free. Gravity muscles, whose effects can only add up to those of gravity, did not exhibit such negative periods (Figure 3C, D). Nor did any muscles for Earth-horizontal movements during which the task requires to compensate for gravity effects (Figure 4). These qualitative results indicate that the presence of negative phasic EMG is specific to the earth-vertical plane and, even more specific, to the timing when gravity can assist movement. Further, it is independent of body-orientation, and both species share it.

Quantification of prevalence, amplitude, and duration of EMG negativity

The vast majority of movements performed in the earth-vertical plane exhibited this general muscular pattern. On average ( \pm SD), across monkeys and humans, the phasic EMG of antigravity muscles exhibited negative values in $90.8 \pm 2.7 \%$ and $72.1 \pm 5.5 \%$ of downward and upward movements, respectively (see Figure 5A and Fig. 5 Suppl. Table 1 for all values). In contrast, in the earth-horizontal plane, rightwards and leftwards movements exhibited negative periods only in $0.014 \pm 0.036 \%$ and $0.021 \pm 0.048 \%$, respectively (see Fig. 5 Suppl. Table 1 for values).

An amplitude index quantified how much gravity force assisted muscle force. This index expressed antigravity muscles activation levels relative to the theoretically required level for exact compensation of gravity effects (the tonic level; see Methods). An index value of $-100 \%$ means utterly relaxed muscles, thus that the gravity force fully participated to arm movement. A value of $0 \%$ means that antigravity muscles precisely compensated for gravity effects, thus that the gravity force did not produce any movement. On average, across monkeys and humans, the amplitude index averaged $( \pm \mathrm{SD})-90.7 \pm 2.8 \%$ and $-66.7 \pm 9.6 \%$ 
195 for downward and upward movements, respectively (see Figure 5B and Fig 5 Suppl. Table 1 196 for all values).

We also computed the duration of negative epochs and expressed it as a percentage of acceleration duration for downward movements and as a percentage of deceleration duration for upward movements. On average $( \pm \mathrm{SD})$, negative periods represented $60.7 \pm 11.5 \%$ of downward acceleration duration and $43.1 \pm 12.4 \%$ of upward deceleration duration (see Figure 5C and Fig 5 Suppl. Table 1).

In both species, the prevalence and extent of negative periods reveal that the brain did not compensate for gravity force. Instead, it lets gravity force much-assisting muscle force in producing arm movements. Next we further demonstrate this by characterizing the temporal organisation of agonist/antagonist muscular activation, which is the most basic and widely used descriptor of muscle patterns (Berardelli et al., 1996; Chiovetto et al., 2010; Cooke and Brown, 1994; Corcos et al., 1989; David et al., 2016; Forgaard et al., 2013; Huang et al., 2015; Irlbacher et al., 2006).

Effect of EMG negativity on muscular activation timing

Figure 6 summarises the activation timings of agonist and antagonist muscles. "Agonist" and "antagonist" are generic denominations that respectively designate the muscles that pull toward or away from the final target. We found that this timing radically changes with movement direction (see Fig. 6 Suppl. Table 1 and 2 for all values and statistical comparisons). Most strikingly, it is the negativity of antagonist muscles (antigravity, open circles) that governed the acceleration of downward movements (Figure 6, blue arrow). During downward movements, both humans and monkeys activated their agonist muscles (gravity muscles, filled squares) nearly at the time of movement initiation. Given electromechanical delays (Cavanagh and Komi, 1979), this agonist activation occurs too late to explain movement initiation. The delayed activation of gravity muscles (agonist) presumably complements the negativity of antigravity muscles (antagonist) to reach the appropriate movement speed (Agostino et al., 1992). The organisation of downward movements is in sharp contrast to earth-horizontal movements, where agonist activation generated acceleration force roughly $100 \mathrm{~ms}$ before movement onset (Figure 6, yellow and green arrows) (Berardelli et al., 1996; Cooke and Brown, 1994; Corcos et al., 1989).

Upward movements (red arrow) exhibited an organisation that was complementary to downward movements. First, the activation of agonist (antigravity) muscles occurred at a similar timing as earth-horizontal movements (filled squares). Then, the agonist (antigravity) 
muscle became negative (open square). This late negativity lets gravity assist muscle force in decelerating the arm upwards. Again, this is in sharp contrast to earth-horizontal movements, where the single activation of antagonist muscles produces the deceleration force. optimization hypothesis according to which the brain takes advantage of gravity effects to discount muscle force that pulls downwards. Next, we directly test this hypothesis by withinmuscle comparison of EMG activity between earth-horizontal and earth-vertical movements.

Within-muscle comparison between earth-horizontal and earth-vertical movements

Humans performed arm movements that were either parallel or perpendicular to the head/feet body axis, from two body orientations (Figure 7A, B). Because the same muscles were responsible for earth-vertical and earth-horizontal movements, one can directly compare the amplitude of muscular activations between movement planes. Specifically, we will compare the activation of gravity muscles in the vertical plane to activation of the same muscles in reciprocal earth-horizontal movements (here reciprocal refers to movements that have the same direction in the ego-centred frame of reference).

For example, in the ego-parallel group (Figure 7A), one can compare the activation of agonist muscles between acceleration phases towards the feet in the two planes (blue vs green targets). If gravity effectively assists muscle force, one would expect reduced activation of gravity muscles in the earth-vertical plane compared to reciprocal movement in the earthhorizontal plane. Following this logic, Figure 7C-F compares muscular activation between reciprocal movements performed in the two planes, for all movement phases that exhibited negativity of antigravity muscles. Data falling below the identity line indicate that earthvertical movements necessitate weaker muscular activations than earth-horizontal ones and vice versa.

Figure 7C-D compares downward movement acceleration to reciprocal movement acceleration in the earth-horizontal plane, for the ego-parallel group and the egoperpendicular group (blue arrows vs green arrows in Figure 7A-B). All individual comparisons fall below the identity line $(n=48)$. This result demonstrates that downwards acceleration required reduced agonist muscle force than horizontal acceleration (all agonist muscles averaged per participant, Student paired two-sided; ego-parallel group, $\mathrm{T}_{7}=6.4$, $\mathrm{P}=0.0004$; ego-perpendicular group, $\mathrm{T} 7=9.6, \mathrm{P}=0.00003$, see bar-graph inserts in Figure $7 \mathrm{C}$ D). Thus, gravity effectively assisted muscle force in accelerating the arm downwards. 
Figure 7E-F compares the decelerations of upward movements and reciprocal movements in the earth-horizontal plane for the two groups (red arrows vs yellow arrows in Figure 7A-B). All but seven individual comparisons fall below the identity line (total $n=48$ ). The acceleration of upward movements required significantly reduced antagonist muscle force (all antagonist muscles averaged, Student paired two-sided; ego-parallel group, $\mathrm{T}_{7}=3.3$, $\mathrm{P}=0.01$; ego-perpendicular group, $\mathrm{T} 7=8.0, \mathrm{P}=0.00009$; see bar-graph inserts in Figure 7E-F). Thus, gravity effectively assisted muscle force in decelerating the arm upwards.

As predicted by the Effort-optimization hypothesis, these results confirm that the negativity of phasic EMGs effectively reduces the muscular effort that pulls downwards in gravity (not body)-coordinates. The neural integration of gravity yields optimal motor planning in both monkeys and humans.

\section{Discussion}

275 Here, we investigated a fundamental question: does the motor system compensate for the gravity force, or does it exploit it as a 'free' force to discount muscle effort during movement? A combination of kinematics and EMG analyses have provided strong support for the Effort-optimization strategy. We analysed multiple variables, but two stand out as most relevant. (1) The relative time to peak velocity unmasked the motor plan at the kinematic level. (2) The negativity of the phasic EMG component revealed the signature of the optimal motor plan where gravity is purposefully exploited for effort-efficient motor control.

As previously demonstrated, shorter acceleration duration and higher peak acceleration for upward than for downward movements is the kinematic signature of optimal integration of gravity into the motor plan (Berret et al., 2008; Gaveau et al., 2014, 2016). If the motor system compensated for the gravity force, kinematics should be directionindependent in the earth-vertical plane, as is the case in the earth-horizontal plane (Gentili et al., 2007; Le Seac'h and McIntyre, 2007). The present results reveal that monkeys produce the same direction-dependent kinematics as humans (Figure 2), suggesting that optimal motor planning in the gravity field may reflect a universal cross-species process.

We sought the neural signature of this optimal motor plan by analysing EMG activity patterns. Our rationale was straightforward. If the brain truly exploits the gravity force to discount muscular effort, specific negative periods should appear in the phasic EMG signal. These negative periods would mean that the muscle produces less force than required to compensate the gravity force, such that the motor system exploits the gravity force to generate movement. We used single-degree-of-freedom movements to systematically vary the effect of 
gravity while the rest of the movement dynamics (inertial forces) remained constant. We 297 found that antigravity muscles exhibited periods of negativity precisely during the acceleration phase of a downward movement and the deceleration phase of an upward movement, when gravity can assist the movement (Figure $3 \& 4$ ). Notably, many studies already observed negativity of the phasic component of muscular activations and forces during vertical movements (Buneo et al., 1994; d'Avella et al., 2006, 2008; Flanders and Herrmann, 1992; Flanders et al., 1994, 1996; Olesh et al., 2017; Russo et al., 2014). However, this phenomenon was primarily ignored and attributed to erratic errors in the separation of noisy signals. The present study demonstrates that negativity is not erratic but systematic.

Furthermore, we demonstrated that the negativity of the phasic component was both consistent and extensive (Figure 5), significantly affected the temporal organisation of the muscle patterns (Figure 6), and decreased the activation of gravity muscles (the muscles pulling with gravity, Figure 7). These results provide strong support for the active participation of the gravity force to movement generation. In both macaques and humans, direction-dependent kinematic and muscular patterns point towards a motor strategy that discounts muscle effort, as previously proposed by computational models (Berret et al., 2008; Crevecoeur et al., 2009; Gaveau et al., 2011, 2014, 2016).

Given the richness of humans' and monkeys' motor repertoires, the study of monoarticular arm movements may seem restrictive. However, it is essential to point out that direction-dependent motor patterns have been observed for movements as varied as monoarticular arm (Crevecoeur et al., 2009; Gaveau and Papaxanthis, 2011; Gaveau et al., 2014, 2016; Gentili et al., 2007; Hondzinski et al., 2016; Sciutti et al., 2012; Le Seac'h and McIntyre, 2007), multi-articular arm (Berret et al., 2008; Papaxanthis et al., 1998a, 1998b; Yamamoto and Kushiro, 2014) and whole-body movements (Manckoundia et al., 2006; Papaxanthis et al., 2003). These results, along with the present findings, provide conceptual support for a general theory that the brain builds internal representations of the environmental and musculoskeletal dynamics to optimise motor planning and control (Franklin and Wolpert, 2011; Guigon et al., 2008; Izawa et al., 2008; Shadmehr and Wise, 2005; Shadmehr et al., 2010; Todorov, 2004; Wolpert and Ghahramani, 2000).

Several studies have provided neurophysiological evidence of gravity internalisation in both macaques and humans (Angelaki et al., 1999, 2004; Indovina et al., 2005; Laurens et al., 2013b, 2013a, 2016; Miller et al., 2008). The present results strongly suggest that a utility

328 of this neural representation of gravity is to discount muscle effort. This optimisation capability seems to be shared by humans and non-human primates as we observed similar 
330 kinematics and EMG patterns in both species. This cross-species neural strategy may 331 underline the fundamental influence of gravity on the evolution, development, and function of 332 motor systems. The metabolic rate was shown to influence body size, resource use, rate of 333 senescence and survival probability (Berghänel et al., 2015; Brown et al., 2004; DeLong et 334 al., 2010; Munch and Salinas, 2009; Strotz et al., 2018; Voorhies and Ward, 1999). Preserving 335 muscle effort may thus represent an essential pursuit for the brain (Baraduc et al., 2013; 336 Bramble and Lieberman, 2004; Carrier et al., 2011; Cheval et al., 2018a, 2018b; 337 Farshchiansadegh et al., 2016; Huang et al., 2012; Inzlicht et al., 2018; Kurzban et al., 2013; 338 Lee et al., 2016; Lieberman, 2015; Mazzoni et al., 2007; Morel et al., 2017; Pageaux, 2016; 339 Pageaux and Gaveau, 2016; Selinger et al., 2015; Shadmehr et al., 2016; Walton et al., 2006; 340 Wang and Dounskaia, 2012). 


\section{Methods}

Monkeys and humans performed standard experiments as previously described elsewhere (monkeys (Moran and Schwartz, 1999; Wang et al., 2010), humans (Gaveau and Papaxanthis, 2011; Gentili et al., 2007; Le Seac'h and McIntyre, 2007)).

Monkey experiments

347 Setup. Three rhesus monkeys (Macaca Mullata; 5.5 to $7.2 \mathrm{~kg}$ ) participated in the study after 348 the approval of all the experimental procedures by the Animal Studies Committee and IACUC. Monkeys were head-fixed and seated in a custom-made primate chair anchored to a virtual reality system. A mirror mounted in front of the monkey's face at an angle of $45^{\circ}$, reflected the display of a monitor. Monkeys wore custom-made glasses (Kodak Wratten filters red \#29 and green \#61), such that visual stimulus rendered in three dimensions as redgreen anaglyphs. Using an optoelectronic tracking system (NDI Optotrak Certus) the 3D position of the monkey's right hand was fed back in real-time on the monitor as a cursor sphere (1 $\mathrm{cm}$ radius). The monkey performed the task using his right arm. A custom-made brace was positioned on the monkey's right arm to restrain the elbow and wrist joints, allowing motion of the arm around the shoulder joint only (Figure 1A).

Task. By operant conditioning, we trained three monkeys to perform fast point-to-point single degree of freedom reaching movements (shoulder rotations) between sets of two targets $(1 \mathrm{~cm}$ radius), from an initial to a final target. We positioned the targets at arm's length. For upward and downward movements (earth-vertical plane), we set-up two targets in a parasagittal plane crossing the centre of rotation of the animal's right shoulder joint. We horizontally aligned the upper target with the animal's shoulder (arm horizontal: $90^{\circ}$ shoulder elevation, $0^{\circ}$ shoulder abduction). We positioned the lower target such that the shoulder was extended by $20^{\circ}$ below horizontal $\left(70^{\circ}\right.$ shoulder elevation, $0^{\circ}$ shoulder abduction). Accordingly, an upward movement consisted of a $20^{\circ}$ shoulder flexion and a downward movement consisted of a $20^{\circ}$ shoulder extension. For rightward and leftward movements, we set-up three targets in a transverse plane crossing the centre of rotation of the animal's shoulders. The central-starting target was the same as the upper target for earth-vertical movements $\left(90^{\circ}\right.$ shoulder elevation,

$3710^{\circ}$ shoulder abduction). We also set-up two additional targets at an angle of $20^{\circ}$ rightward $372\left(90^{\circ}\right.$ shoulder elevation, $20^{\circ}$ shoulder abduction) and $20^{\circ}$ leftward $\left(90^{\circ}\right.$ shoulder elevation, $37320^{\circ}$ shoulder adduction), taking as reference the horizontal position of the arm on the central 
target. A rightward movement consisted of a $20^{\circ}$ abduction (starting at the central and ending at the most rightward target), and a leftward movement consisted of a $20^{\circ}$ adduction (starting at the central and ending at the most leftward target). Monkeys performed 200 to 300 trials per sessions (total number of trials recorded for each monkey: $\mathrm{D}=2854 ; \mathrm{J}=2347 ; \mathrm{Z}=2987$ ).

Kinematics recording. We used an optoelectronic tracking system (NDI Optotrak Certus, $200 \mathrm{~Hz}$ ) to record the 3D position of infrared emitting diodes (markers) taped on the animal arm and brace. The most distal marker was used to provide the hand position feedback to the monkey.

Electromyographic recording. We recorded EMG activity with pairs of insulated singlestranded stainless steel wires (A-M SYSTEMS, 790700). Before the experiment, we inserted two twisted wires ( $3 \mathrm{~mm}$ un-insulated at their ends) in each targeted muscle ( $1 \mathrm{~cm}$ separation) using 33G hypodermic needles (TSK STERiJECT; for further details, see (Kurtzer et al., 2006; Moran and Schwartz, 1999)). We plugged the wires into a custom-made printed circuit board, itself linked to a differential EMG amplifier (GRASS TECHNOLOGIES, QP511). Then, to verify the appropriate positioning of each electrode, we induced muscle twitch using microstimulations. The Optotrak (ODAU, NDI) sampled the raw EMG activity at $4 \mathrm{kHz}$, synchronously with kinematic signals. We recorded activations patterns of the following eleven muscles: Deltoids (Anterior, Middle and Posterior), Triceps (long and lateral heads), Biceps (two heads), Pectoralis Major (Upper and Lower), Latissimus Dorsi and Infraspinatus.

\section{Human experiments}

Sixteen participants ( 4 females; mean age $=25.4 \pm 5.5 \mathrm{y}$; mean weight $=74.2 \pm 9.6 \mathrm{~kg}$; mean height $=168 \pm 32 \mathrm{~cm}$ ) voluntarily participated in the experiments. All participants were right-handed (Edinburg Handedness Inventory; Oldfield, 1971), with normal or corrected to normal vision and did not have any neurological or muscular disorders. The local ethics committee approved the experimental protocol that was carried out in agreement with legal requirements and international norms (Declaration of Helsinki, 1964).

404 Experimental protocol. Participants performed single-degree-of-freedom arm movements with their fully extended arm (shoulder rotations). We randomly separated the sixteen participants into two groups of eight each. Participants from the "ego-parallel" group performed arm movements in the parasagittal plane crossing their right shoulder (Figure 1B). 
408 Participants from the "ego-perpendicular" group performed arm movements in the transversal 409 plane crossing their right shoulder (Figure 1C). For each participant, we positioned three 410 targets in the movement plane corresponding to the respective group. For both groups, the 411 central target implied a $90^{\circ}$ shoulder elevation and a $0^{\circ}$ shoulder abduction (arm 412 perpendicular to the trunk; dark arm in Figure 1B-C). From this central target, the other two 413 targets triggered $20^{\circ}$ shoulder rotations that were opposite in the plane of motion (light arms 414 in Figure 1B-C).

Participants performed arm pointing movements in two conditions of body orientation 416 (Figure 1B-C). In one condition, they sat upright with their head-feet body axis parallel to 417 gravity. In the other condition, they were $90^{\circ}$ rotated in roll and lied on their left side with the 418 head-feet body axis perpendicular to gravity. Between body-orientation conditions, target 419 position was kept constant in the participant egocentric frame of reference (targets rotated 420 with the participant). Half of the experiment consisted of arm movements that were parallel to 421 the gravity vector (seating upright in the ego-parallel group, and lying on the side in the ego422 perpendicular group). The other half consisted of arm movements that were perpendicular to 423 it (lying on the side in the ego-parallel group, and seating upright in the ego-perpendicular group). We counterbalanced the order of body-orientation conditions between participants.

We instructed participants to perform accurate and uncorrected arm movements at fast speed. Before the experiment, participants performed as much practice trials as they wanted in order to familiarise with the task. Then, for each body orientation, participants performed 60 trials (30 in each direction) in a randomized order (30 trials $* 2$ body orientations $* 2$ directions $* 16$ participants $=1920$ trials). Each trial took place as follows. The participant pointed at the central target and held it. After a random delay time ( 1 to $2 \mathrm{~s}$ ), he/she received instructions about which target to point. The participant performed the movement and held the final position until the experimenter stopped the recording and informed him/her to relax.

Kinematics recording. We used an optoelectronic tracking system (Vicon, Oxford, UK, eight cameras, $200 \mathrm{~Hz}$ ) to record the position five reflective markers taped on the participants' arm: shoulder (acromion), elbow (lateral epicondyle), wrist (between the cubitus and radius styloid processes), hand (first metacarpophalangeal joint), and the nail of the index fingertip. 
twelve muscles: Deltoids (Anterior, Middle and Posterior), Triceps (long and lateral head), Biceps (two heads), Brachioradialis, Infraspinatus, Pectoralis Major (Upper and lower heads), and Latissimus dorsi.

Data analysis

We processed kinematic and EMG data using custom MATLAB scripts (MathWorks). As monkey and human data were processed similarly, we describe the data analysis for both experiments in a single section.

Kinematics. Kinematic data processing was similar to previous studies (Gaveau and Papaxanthis, 2011; Gaveau et al., 2014). We filtered the position (low-pass, 5Hz cut-off, fifthorder, zero-phase distortion, "butter" and "filtfilt" functions) before differentiation. A 10\% threshold of the peak angular velocity defined movement onset and offset. We rejected from further analyses trials where the velocity profile presented more than one local maxima (on average $<5 \%$ of trials in monkeys and $<2 \%$ of trials in humans). We then calculated the following parameters (Fig. 2 Suppl. Figure 1A): movement duration (MD); movement amplitude; peak acceleration (PA) and relative duration to peak acceleration (rDPA $=$ duration to peak acceleration / MD); peak velocity (PV) and relative duration to peak velocity (rDPV= duration to peak velocity / MD). We also computed angular joint displacements to control that shoulder internal/external rotations, as well as wrist or elbow rotations, were negligible.

$E M G$. We first rectified and filtered EMG signals (bandpass $20-300 \mathrm{~Hz}$, third-order, zerophase distortion, "butter" and "filtfilt" functions). Then we integrated this signal over $5 \mathrm{~ms}$ bins and cut it off $500 \mathrm{~ms}$ before movement onset and $500 \mathrm{~ms}$ after movement offset. To compare EMGs between muscles, participants, and datasets, we normalised each trace by the maximum value observed for the corresponding muscle in the dataset. We then averaged trials across three repetitions, resulting in 10 averaged trials to be analysed.

We used a well-known subtraction procedure that was proposed to isolate the phasic

471 Flanders and Herrmann, 1992; Flanders et al., 1994, 1996; Olesh et al., 2017; Prange et al., 472 2009b, 2012; Russo et al., 2014). We computed the average values of the integrated EMG 473 signals from $1 \mathrm{~s}$ to $0.5 \mathrm{~s}$ before movement onset and from $0.5 \mathrm{~s}$ to $1 \mathrm{~s}$ after movement offset 474 (Fig. 2 Suppl. Figure 2). We used these average values to compute the tonic component as a 
475 linear interpolation between them. Finally, we computed the phasic component by subtracting 476 the tonic component from the full integrated EMG signal.

477 We quantified the negativity of the phasic muscular activations by calculating the

478 following parameters: i) the duration of the negative epoch, defined as the time interval where 479 the phasic activity dropped below zero minus the 95\% confidence interval (computed on the 480 integrated EMG signals from $1 \mathrm{~s}$ to $0.5 \mathrm{~s}$ before movement onset) and this for longer than $48140 \mathrm{~ms}$; ii) an index of the amplitude of the negative epoch, computed as follows:

$482 \quad$ PminT $\times 100$

483 where Pmin is the phasic maximally negative value (during the negative epoch), and $\mathrm{T}$ is the tonic value subtracted at the time of Pmin. An amplitude index value of $-100 \%$ means that muscles were completely relaxed and that the gravity force fully participated in generating the arm motion. A value of $0 \%$ means that antigravity muscles precisely compensated for gravity force; i.e., that gravity did not produce any arm motion; iii) the frequency with which a negative phase was detected amongst all trials.

We also characterized muscular activation using the following parameters: i) the onset 490 of muscle activation, defined as the time where the phasic activity first rose above zero plus 491 the $95 \%$ confidence interval (computed on the integrated EMG signals from $1 \mathrm{~s}$ to $0.5 \mathrm{~s}$ before 492 movement onset) for longer than $40 \mathrm{~ms}$; v) the mean normalised integrated signal over the 493 acceleration period (from movement onset minus $100 \mathrm{~ms}$ to time to peak velocity minus 494 100ms); vi) the mean normalised integrated signal over the deceleration period (from the time 495 to peak velocity minus $100 \mathrm{~ms}$ to movement offset minus $100 \mathrm{~ms}$ ).

\section{Statistics}

498 We checked that all variables were normally distributed (Shapiro-Wilk W test) and 499 that their variance was equivalent (Mauchly's test). We used repeated measure ANOVA, 500 applied to means of separate sessions in each monkey, as well as mean values for each human 501 subject. Post-hoc comparisons were performed using Scheffé tests. Student paired two-sided 502 tests were used to compare muscle activation levels between body orientations in humans. In 503 all cases, the level of significance was equal to 0.05 . 


\section{$504 \quad$ Figures \& Legends}

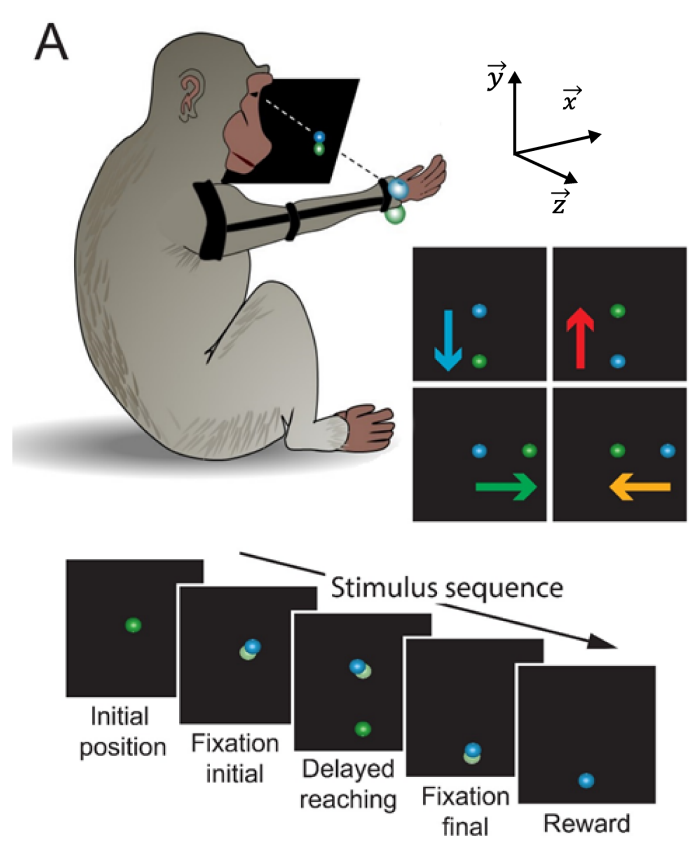

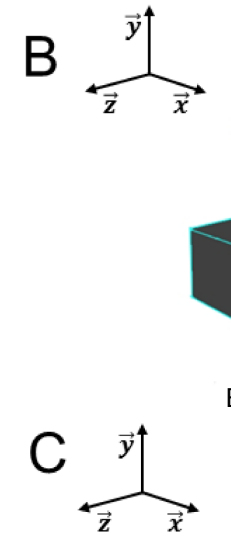

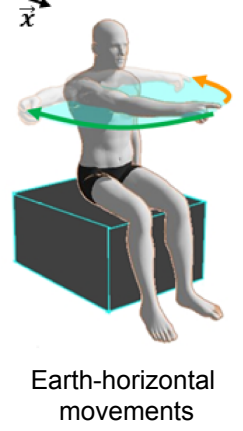

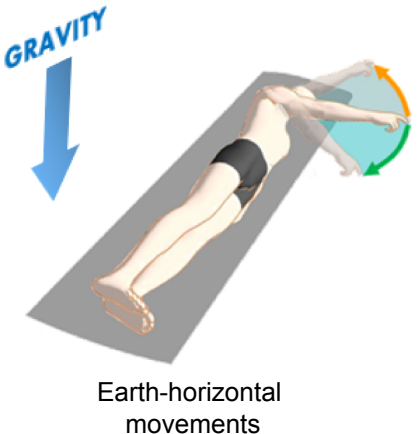

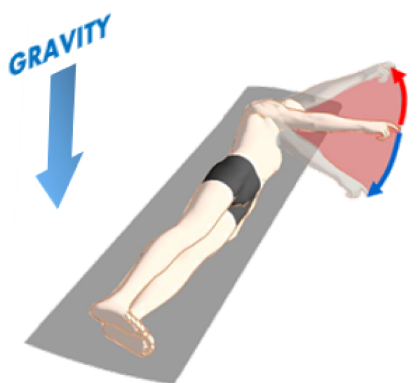

Earth-vertical movements

506 Figure 1. Experimental setup. A. Illustration of the monkey task. Using a virtual reality 507 environment, three monkeys performed mono-articular point-to-point arm movements 508 (shoulder rotations, a brace restraining elbow and wrist joints) between sets of two targets. 509 Positions of initial and final targets implied a leftward (yellow arrow), a rightward (green), a 510 downward (blue) or an upward (red) movement. The monkeys performed a delayed-reaching 511 task with fixation periods on the initial and the final target. The lower part of the panel depicts 512 the stimulus sequence. First, the initial target appeared and its colour changed to light green 513 when the monkey's hand touched it. After a random delay time, the final target appeared, and 514 the monkey had to reach it as soon as the initial target disappeared (additional random delay 515 time). Finally, the monkey obtained a liquid reward after remaining stationary on the final 516 target for another random delay time. B. Illustration of the human task for the ego-parallel 517 group. Starting with their right arm perpendicular to the trunk (dark middle arm), height 518 humans performed single-degree-of-freedom reaching arm movements (shoulder rotations) 519 between sets of two targets. Positions of targets implied movements toward the head 520 (shoulder flexion) or the feet (shoulder extension). Participants experienced two conditions of 521 body orientations to record movements in the earth-vertical plane (participant seated, red/blue 522 arrows) and in the earth-horizontal plane (participant reclined, yellow/green arrows); i.e. 
523 targets were rotated with the participant. C. Illustration of the human task for the ego524 perpendicular group (height other human participants, same organisation as the first group, 525 see methods). The positions of targets implied movements toward the right side of the body 526 (shoulder abduction) or the left side of the body (shoulder adduction). Participants 527 experienced two conditions of body orientations to record movements in the earth-vertical 528 plane (participant reclined, red/blue arrows) and in the earth-horizontal plane (participant 529 seated, yellow/green arrows); i.e. targets rotated with the participant. In all three panels, the $\mathrm{x}$, $530 \mathrm{y}, \mathrm{z}$ axes illustrate the world coordinate system. For all figures, the colour-code is gravity531 centred, as follows: red is against gravity (towards positive Y-axis); blue is with gravity 532 (negative Y), yellow is perpendicular to gravity, leftwards (negative Z); green is 533 perpendicular to gravity, rightwards (positive Z). 
Earth-vertical plane
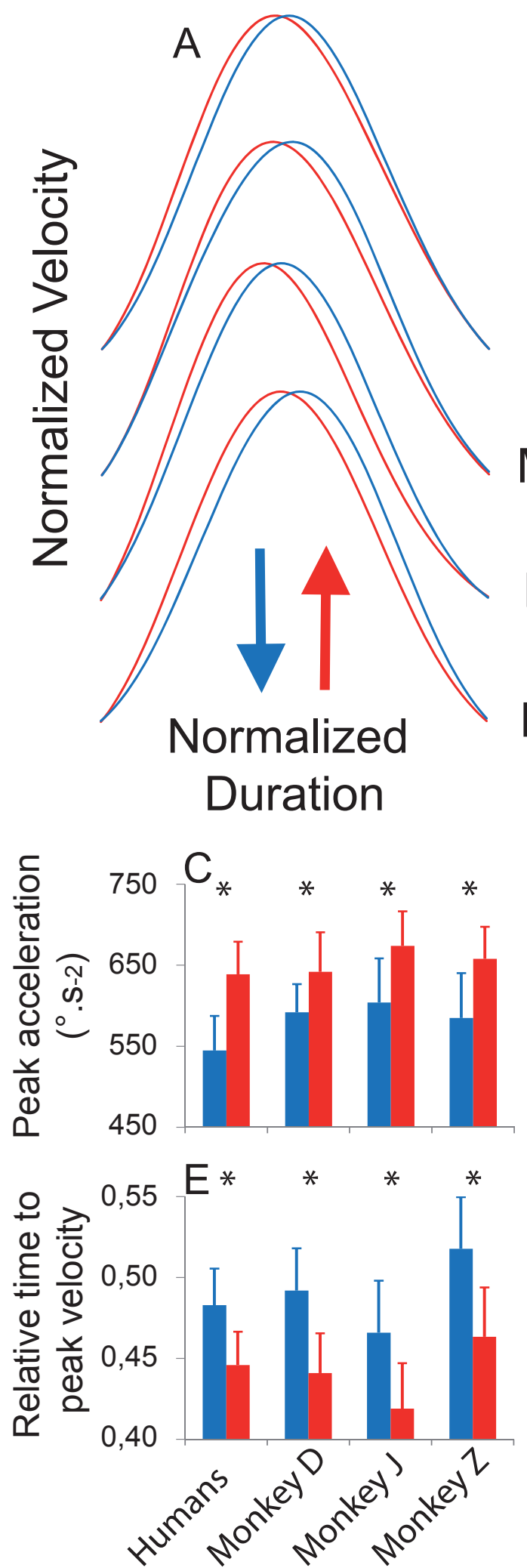

Earth-horizontal plane
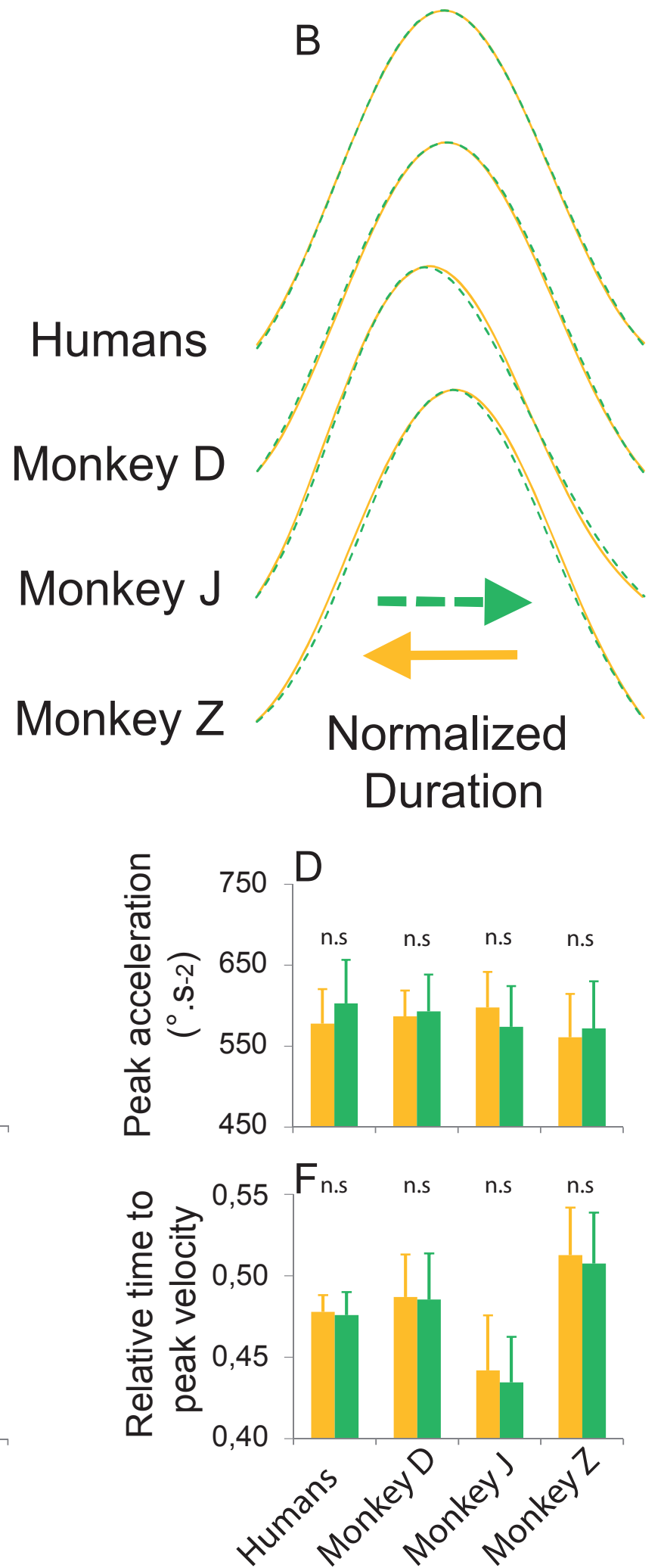

535 Figure 2. Similar direction-dependent arm kinematics in monkeys and humans. Mean

536 velocity profiles recorded in opposed directions in the earth-vertical (A) and the earth- 
537 horizontal plane (B) for each monkey and all humans $(n=16)$. Coloured arrows indicate 538 movement directions. Traces are amplitude and duration normalised to ease directional 539 comparisons. Direction-dependent kinematics is observed in the earth-vertical plane, but not 540 in the earth-horizontal plane. The amplitude of the acceleration peak for each monkey and all 541 humans $(n=16)$ is presented for opposed directions in the earth-vertical plane $(\mathbf{C})$ and the 542 earth-horizontal plane (D). The relative duration to peak velocity for each monkey and all 543 humans $(\mathrm{n}=16)$ is presented for opposed directions in the earth-vertical plane $(\mathbf{E})$ and the 544 earth-horizontal plane (F). Error bars represent the standard deviation of the mean between 545 recording sessions for each monkey and between participants for humans. 


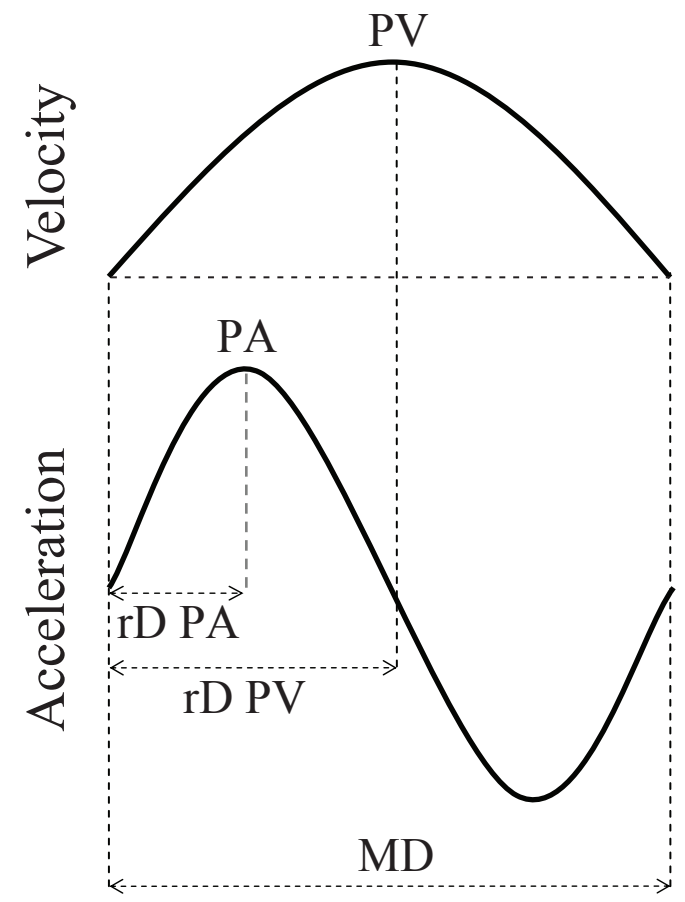

547 Figure 2 Supplemental Figure 1. Illustration of kinematic analysis. Kinematic parameters 548 computed on velocity and acceleration profiles (see methods). MD, movement duration; PA, 549 peak acceleration; PV, peak velocity; rDPA, relative duration to peak acceleration; rDPV, 550 relative duration to peak velocity. 


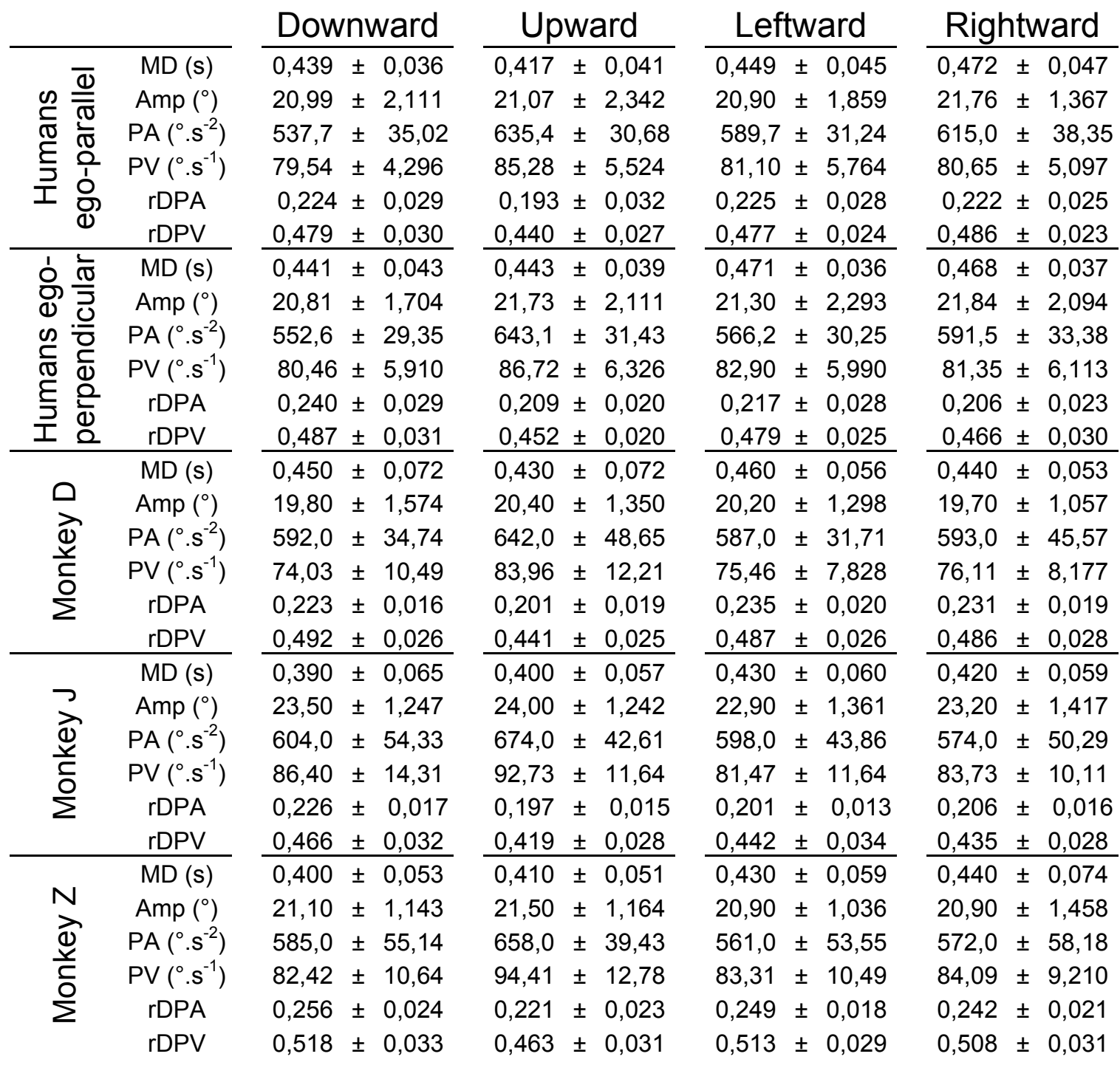

552 Figure 2 Supplemental Table 1. Kinematic parameters describing arm movements.

553 Mean values $( \pm \mathrm{SD})$ recorded for each monkey, and both groups of humans $(n=8$ in each

554 group) are presented for downward, upward, leftward and rightward movements. MD,

555 movement duration in second (s); Amp, amplitude in degree $\left(^{\circ}\right)$; PA, peak acceleration in 556 degree per squared second $\left({ }^{\circ} \cdot \mathrm{s}^{-2}\right)$; PV, peak velocity in degree per second $\left({ }^{\circ} \cdot \mathrm{s}^{-1}\right)$; $\mathrm{rDPA}$, 557 relative duration to peak acceleration (duration to peak acceleration / movement duration); $558 \mathrm{rDPV}$, relative duration to peak velocity (duration to peak velocity / movement duration). 


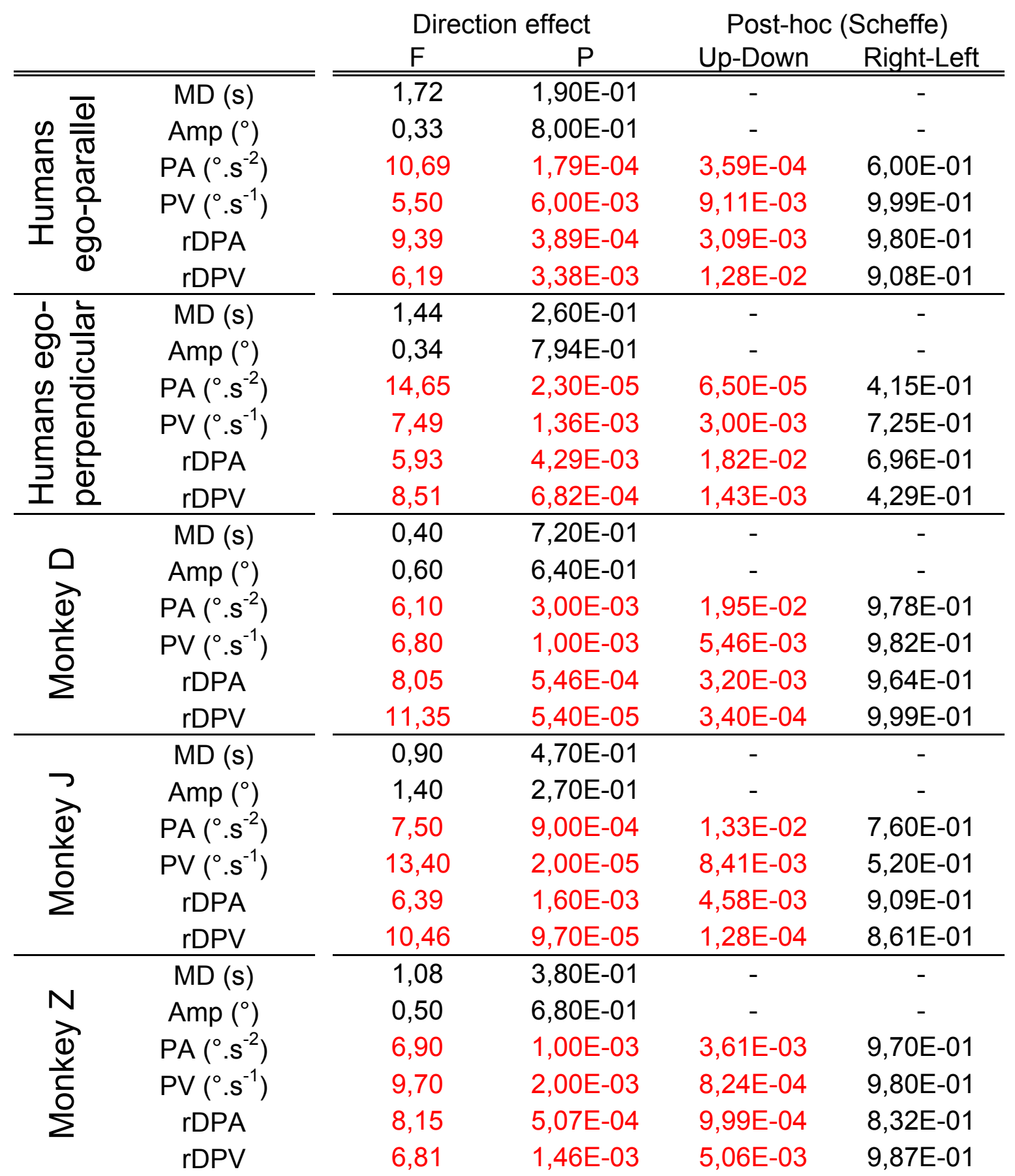

560 Figure 2 Supplemental Table 2. Statistical comparisons on kinematic parameters. F and

$561 \mathrm{P}$ values resulting from an ANOVA test on direction effect are presented for all parameters 562 presented in Figure 2 Suppl. Table 1. Results of post-hoc analyses (Scheffe) on opposed 563 directions in the two planes (Up vs Down \& Right vs Left) are presented for parameters on 564 which we found a significant effect of direction $(\mathrm{p}<0.05)$. Like humans, monkeys exhibit 565 directional asymmetries in the earth-vertical plane but not in the earth-horizontal plane, 566 suggesting an optimal integration of gravity effect for the minimisation of muscular effort. 


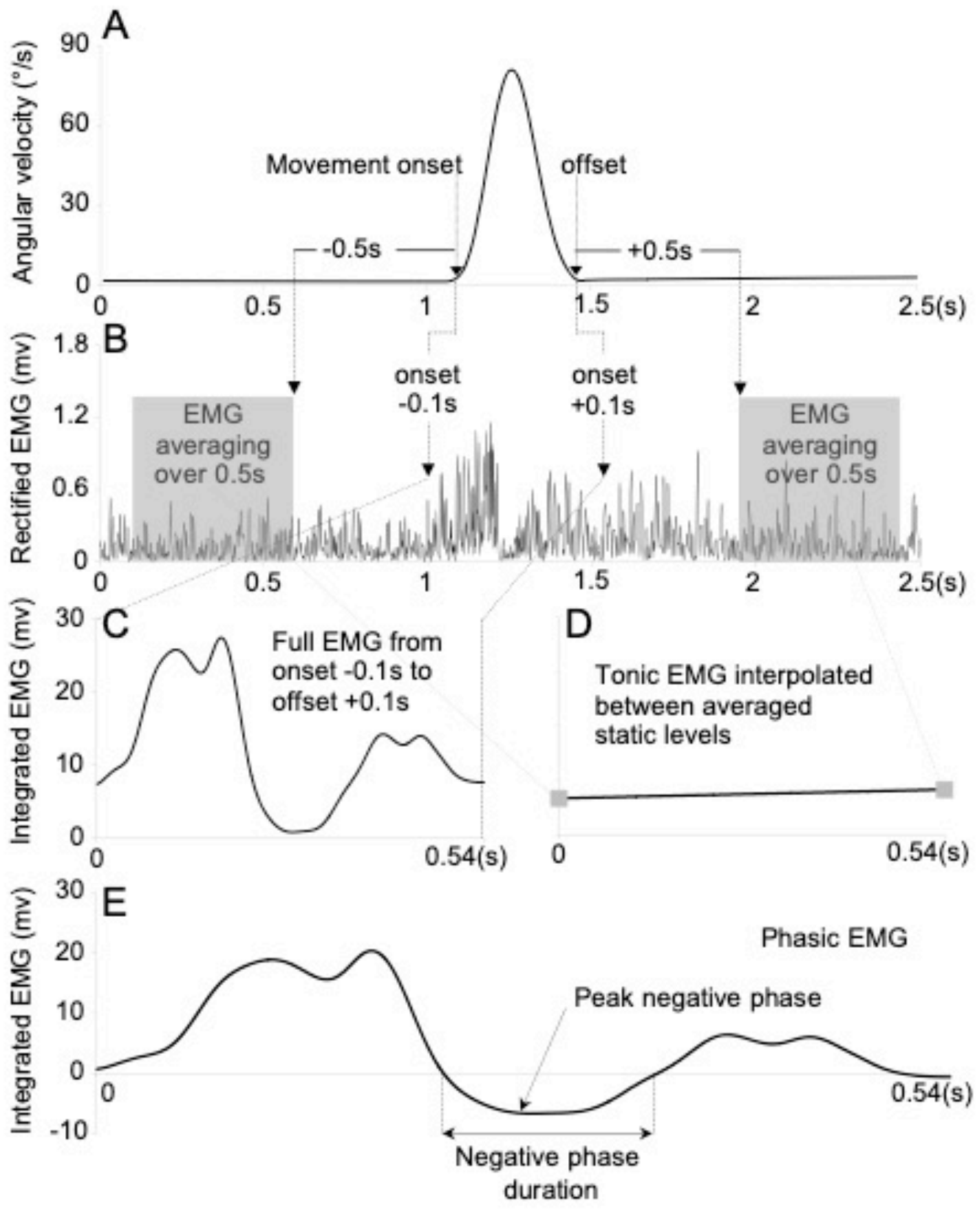

568 Figure 2 Supplemental Figure 2. Illustration of EMG analysis. Separation procedure of

569 the tonic and phasic component of the EMGs (see methods). A. Movement onset and offset 570 were detected on the velocity profile. B. Timings of interest were synchronically detected on 571 EMG data around movement onset and offset: the time intervals when the participant was 572 waiting on the initial target (most leftward grey window) and the final target (most rightward 573 grey window). C. Integrated full EMG corresponding to the movement of the arm (from $574100 \mathrm{~ms}$ before movement onset to, to $100 \mathrm{~ms}$ after movement offset). D. Tonic EMG 
575 component obtained by linear interpolation between the integrated values recorded when the 576 participant was waiting on the initial and final targets; i.e. first and last values in panel D are 577 obtained by integrating signals from the first and second grey windows in panel B. E. Phasic 578 EMG component obtained by subtracting the tonic component (D) from the full EMG (C). 


\section{Movement direction}

\section{Downwards Upwards}

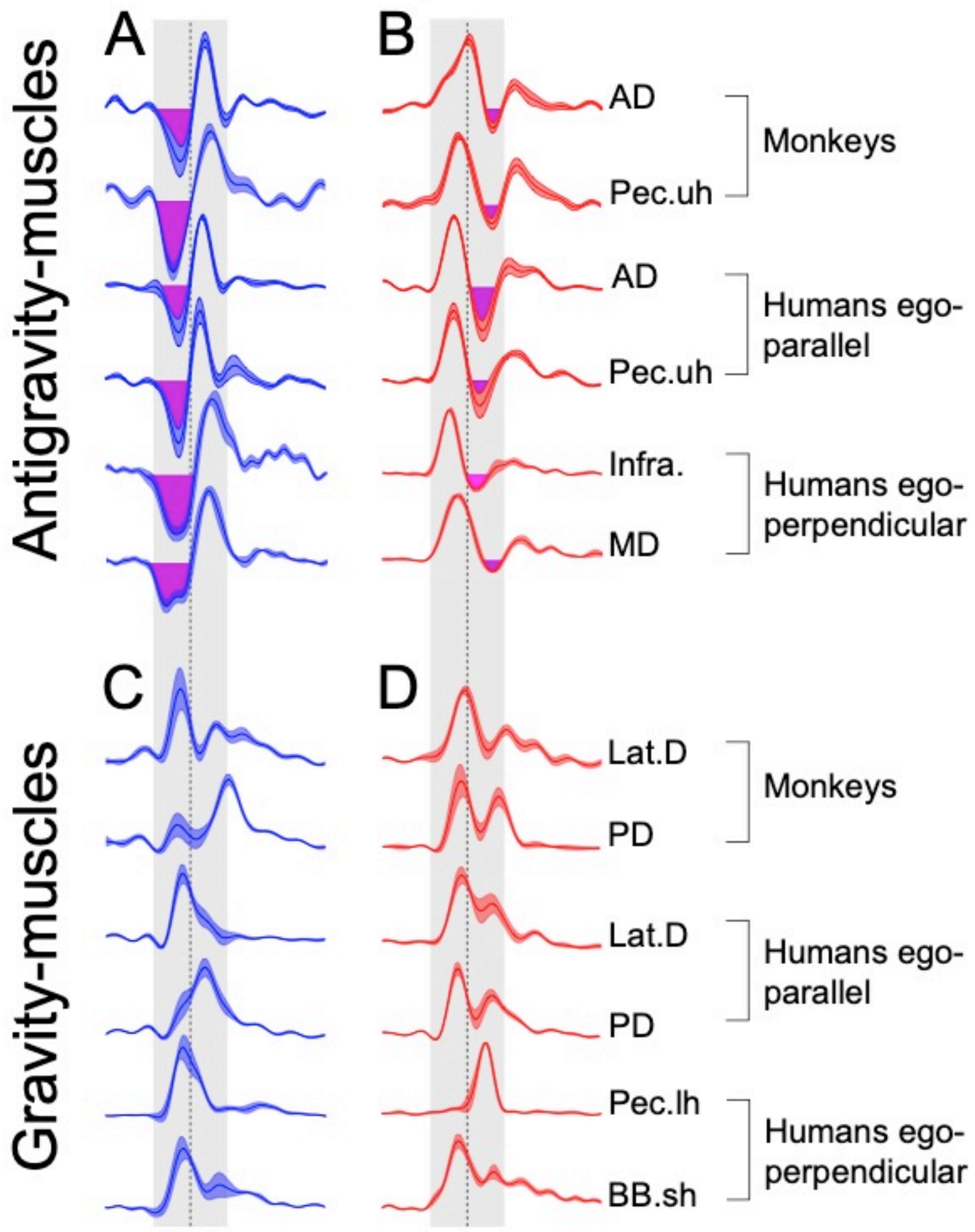

580 Figure 3. Phasic EMG patterns of earth-vertical movements reveal gravitational force

581 contribution to the motion of the arm. Mean ( \pm SE) phasic EMGs recorded in monkeys 
$582(\mathrm{n}=3)$ and both groups of humans ( $\mathrm{n}=8$ in each group) during earth-vertical movements. Left

583 column (panels A and C, blue traces) presents EMGs recorded during downward movements

584 while right column (panels B and D, red traces) presents EMGs recorded during upward 585 movements. Upper row (panels A and B) presents EMG activations of antigravity muscles 586 (pulling upwards, against gravity): Anterior Deltoid (AD), upper head of Pectoralis major 587 (Pec. uh), Infraspinatus (Infra.), Middle Deltoid (MD). Those muscles pull against gravity, i.e. 588 away from the final target during a downward movement and towards the final target during 589 an upward movement. Lower row (panels C and D) presents EMG activations of gravity 590 muscles (pulling downwards, with gravity): Latissimus dorsi (Lat.D), Posterior Deltoid (PD), 591 lower head of Pectoralis major (Pec.lh), the short head of Biceps Brachii (BB.sh). Those 592 muscles pull with gravity; i.e. towards the final target during a downward movement and 593 away from the final target during an upward movement. Purple Area denotes phases where 594 epochs of negativity were detected (see Methods). Such epochs were precisely observed for 595 antigravity muscles (Panels A and B) during the acceleration of a downward movement and 596 during the deceleration of an upward movement; i.e., when gravity effects can help the 597 muscle and therefore discount muscle effort. EMG traces were aligned on movement onset 598 and normalised in duration and amplitude before averaging between participants. The grey 599 earth-vertical area denotes movement duration (shifted 100ms backwards to account for the 600 electromechanical delay), and the dashed earth-vertical line denotes $50 \%$ of movement 601 duration. 


\section{Movement direction}

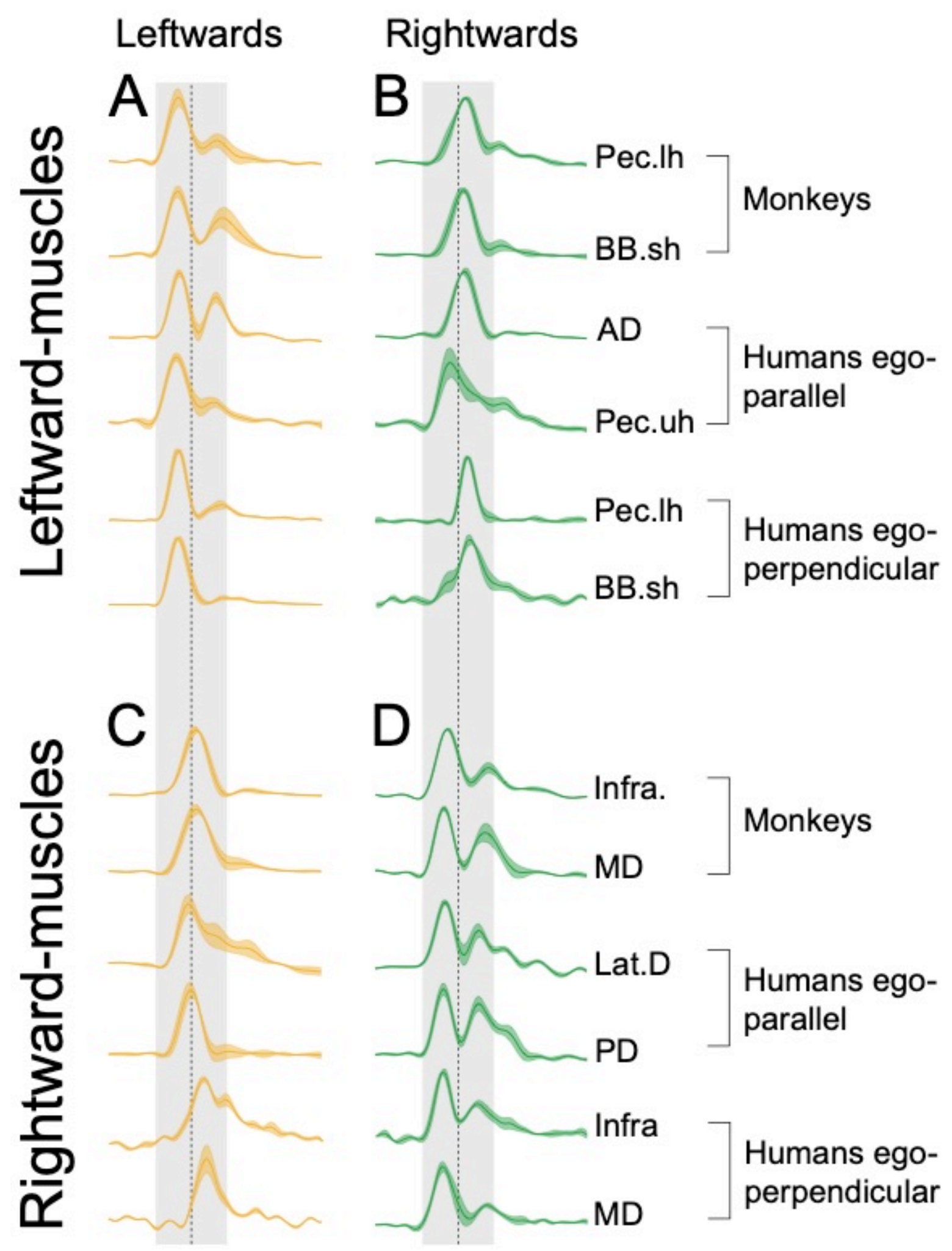

603 Figure 4. Phasic EMG patterns of earth-horizontal movements. Same layout as Figure 3. 604 Mean $( \pm$ SE) phasic EMGs are presented for monkeys $(n=3)$ and both groups of humans $(n=8$ 
605 in each group). Left column (panels A and C, yellow traces) presents EMGs recorded during 606 leftward movements, and right column (panels B and D, green traces) presents EMGs 607 recorded during rightward movements. Upper row (A and B) presents EMG activations of 608 muscles pulling leftwards. Those muscles pull perpendicularly to gravity, i.e. away from the 609 final target during a rightwards movement and towards the final target during a leftward 610 movement. Lower row (C and D) presents EMG activations of muscles pulling rightwards. 611 Those muscles also pull perpendicularly to gravity; i.e. towards the final target during a 612 rightward movement and away from the final target during a leftward movement. No negative 613 periods were detected during earth-horizontal movements, meaning that gravity effects 614 (perpendicular to movement direction) were correctly compensated. EMG traces were aligned 615 on movement onset and normalised in duration and amplitude before averaging between 616 participants. The grey area denotes movement duration (shifted $50 \mathrm{~ms}$ backwards to account 617 for the electromechanical delay), and the dashed earth-vertical line denotes $50 \%$ of 618 movement duration. 

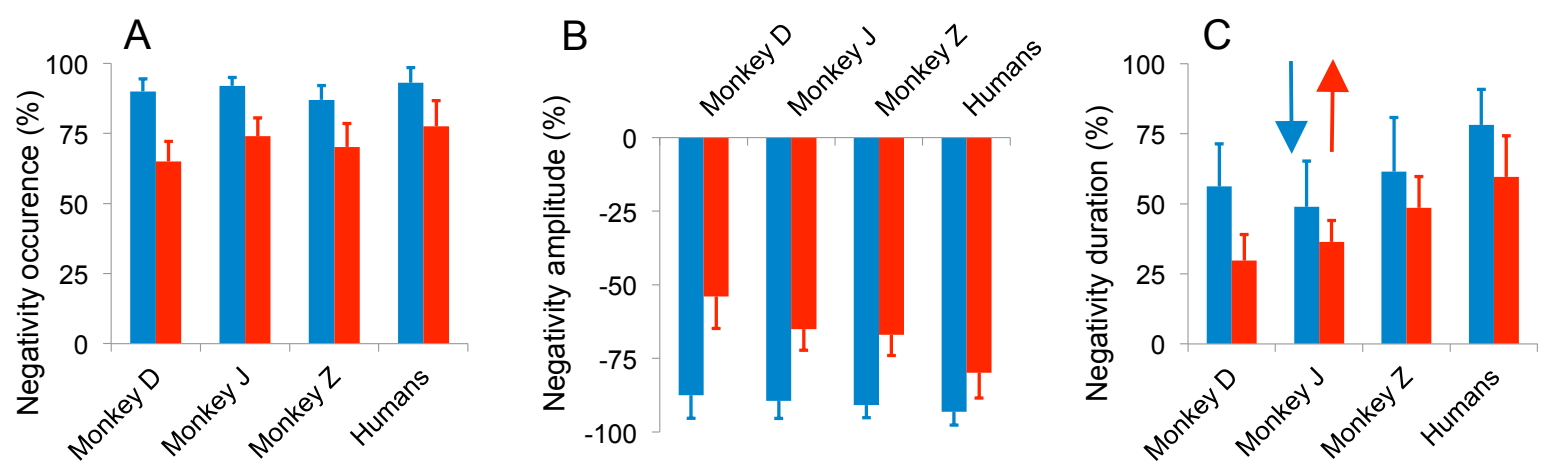

620 Figure 5. Substantial negativity of phasic EMGs. Characterisation of negative epochs

621 recorded for antigravity muscles (the muscles pulling against gravity, presented in panels A

622 and B of Figure 3) during earth-vertical movements. Mean values ( \pm SD) are presented for

623 each monkey and all humans $(\mathrm{n}=16)$. Coloured arrows indicate movement directions (blue for

624 downwards; red for upwards). A. Mean occurrence percentage (number of positive

625 detection/number of trial) of a negative phase for each monkey and humans. B. Maximal

626 negativity amplitude was expressed as a percentage of the subtracted tonic value (see

627 Methods). A value of $-100 \%$ means the muscle was utterly relaxed. Thus gravity helped to

628 move the arm. On the contrary, a value of $0 \%$ means gravity effects were correctly

629 compensated for by muscle force. C. Mean negative phase duration expressed as a percentage

630 of the acceleration duration for downward movement and as a percentage of the deceleration

631 duration for downward movements. Error bars represent the standard deviation of the mean

632 between recording sessions for each monkey and between participants for humans. 


\begin{tabular}{|c|c|c|c|c|c|c|c|}
\hline & & Down & Up & & eft & & ight \\
\hline $\mathscr{\mathscr { D }} \stackrel{\bar{\Phi}}{=}$ & Occurrence (\%) & $94,3 \pm 5,38$ & $79,2 \pm 12,1$ & 0,01 & $\pm 0,01$ & 0,02 & $\pm 0,00$ \\
\hline E $\frac{0}{\partial} \frac{0}{0}$ & Amplitude (\%) & $-95,1 \pm 4,61$ & $-80,7 \pm 10,6$ & - & \pm & - & \pm \\
\hline & Duration (\%) & $76,1 \pm 14,7$ & $57,6 \pm 16,6$ & - & \pm & - & \pm \\
\hline 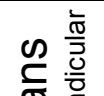 & Occurrence (\%) & $91,8 \pm 4,34$ & $76,2 \pm 9,9$ & 0,02 & $\pm 0,01$ & 0,01 & $\pm 0,01$ \\
\hline 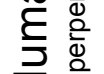 & Amplitude (\%) & $-91,0 \pm 5,16$ & $-78,7 \pm 8,1$ & - & \pm & - & \pm \\
\hline 工 8 & Duration (\%) & $80,1 \pm 13,9$ & $61,5 \pm 14,6$ & - & \pm & - & \pm \\
\hline$\stackrel{0}{2}$ & Occurrence (\%) & $89,6 \pm 4,40$ & $64,8 \pm 7,24$ & 0,03 & $\pm 0,01$ & 0,03 & $\pm 0,01$ \\
\hline 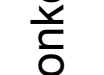 & Amplitude (\%) & $-87,5 \pm 7,83$ & $-53,9 \pm 10,8$ & - & \pm & - & $\pm \quad-$ \\
\hline$z$ & Duration (\%) & $56,2 \pm 15,2$ & $29,7 \pm 9,3$ & - & \pm & - & \pm \\
\hline$\vec{d}$ & Occurrence (\%) & $91,7 \pm 3,34$ & $74,4 \pm 6,59$ & 0,01 & $\pm 0,01$ & 0,02 & $\pm 0,01$ \\
\hline 동 & Amplitude (\%) & $-89,4 \pm 6,01$ & $-65,2 \pm 7,24$ & - & \pm & - & $\pm \quad-$ \\
\hline & Duration (\%) & $48,9 \pm 16,3$ & $36,4 \pm 7,66$ & - & \pm & - & \pm \\
\hline$\stackrel{N}{\lambda}$ & Occurrence (\%) & $86,7 \pm 5,10$ & $70,2 \pm 8,30$ & 0,01 & $\pm 0,01$ & 0,02 & $\pm 0,01$ \\
\hline$\frac{r}{c}$ & Amplitude (\%) & $-90,9 \pm 4,28$ & $-67,4 \pm 7,02$ & - & \pm & - & \pm \\
\hline & Duration (\%) & $61,5 \pm 19,4$ & $48,5 \pm 11,2$ & - & \pm & - & \pm \\
\hline
\end{tabular}

634 Figure 5 Supplemental Table 1. Characterisation of the negativity of the phasic

635 component. Mean values $( \pm \mathrm{SD})$ recorded for each monkey, and both groups of humans $(\mathrm{n}=8$

636 in each group) are presented for downward and upward movements. Occurrence: percentage

637 of trials where a negative phase was detected. Amplitude: percentage-ratio of negativity min

638 value detected on the phasic component over the tonic value that was subtracted at the same

639 time. Duration: percentage-ratio of the negative phase duration over the acceleration duration

640 and the deceleration duration for downward and upward movements, respectively. 


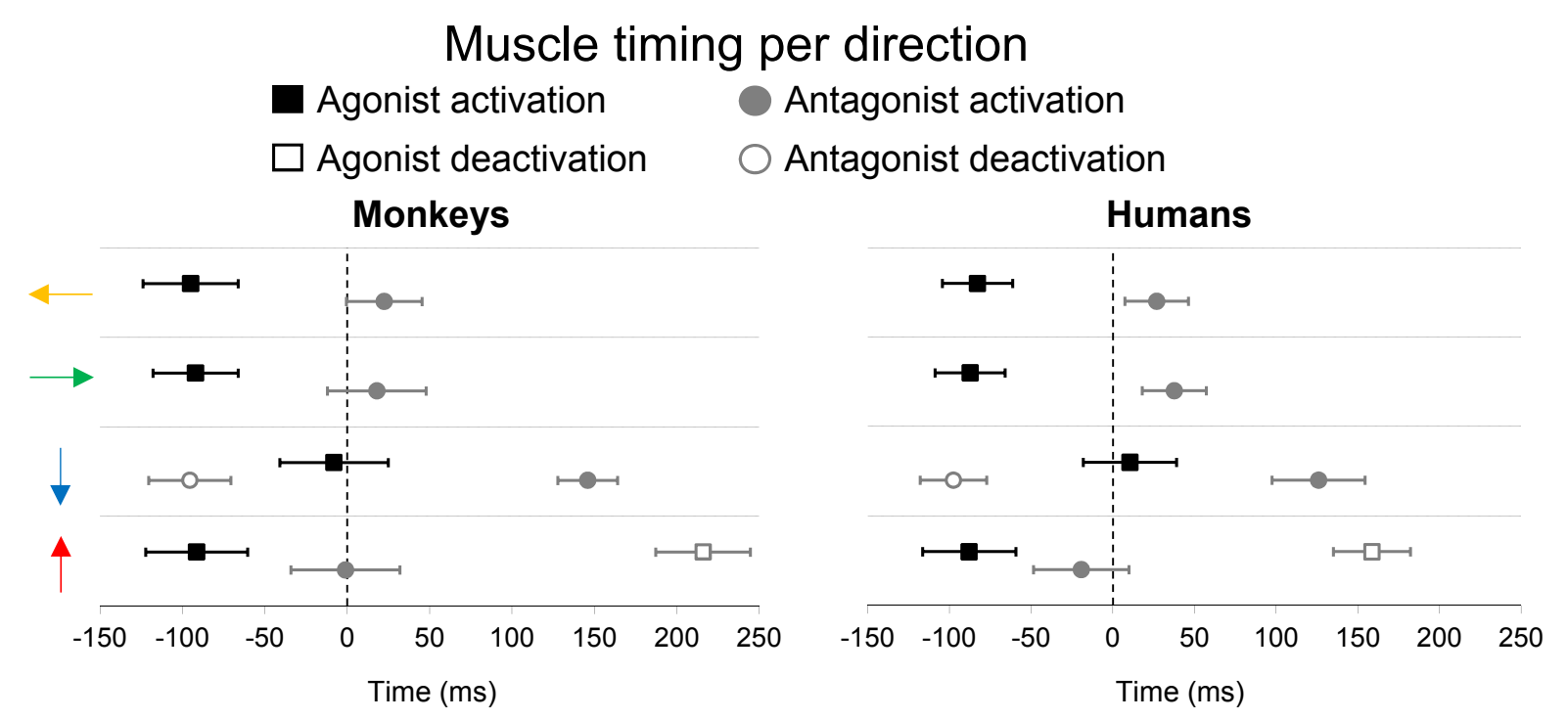

641 Fig 6. Gravity shapes the temporal organisation of muscular activation patterns. Mean

642 ( \pm SD) onset timings (see methods) of agonist and antagonist muscles activations for 643 monkeys (left panel, $n=3$ ) and humans (right panel, $n=16$ ). "Agonist" and "antagonist" are 644 generic denominations that respectively designate the muscles that pull toward or away from 645 the final target. Muscular activity recorded in each movement direction are presented in 646 separate rows (coloured arrows indicate movement direction). EMG traces were aligned on 647 movement onset. 


\begin{tabular}{|c|c|c|c|c|c|}
\hline & & Down & Up & Left & Right \\
\hline \multirow{3}{*}{ 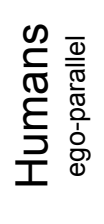 } & Agonist (ms) & $9,21 \pm 24,2$ & $-90,0 \pm 28,2$ & $-84,4 \pm 22,8$ & $-87,7 \pm 19,8$ \\
\hline & Antagonist (ms) & $128 \pm 28,2$ & $-15,7 \pm 29,0$ & $29,2 \pm 20,4$ & $38,6 \pm 20,9$ \\
\hline & Negativity (ms) & $-89,0 \pm 20,4$ & $154 \pm 23,5$ & $-\quad \pm \quad-$ & $-\quad \pm \quad-$ \\
\hline \multirow{3}{*}{ 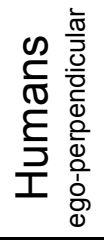 } & Agonist (ms) & $12,1 \pm 32,9$ & $-85,7 \pm 29,0$ & $-81,5 \pm 20,2$ & $-86,7 \pm 23,1$ \\
\hline & Antagonist (ms) & $124 \pm 29,4$ & $-22,6 \pm 27,6$ & $27,9 \pm 18,0$ & $36,2 \pm 19,9$ \\
\hline & Negativity (ms) & $-106 \pm 18,8$ & $163 \pm 29,6$ & $-\quad \pm$ & $\pm \quad-$ \\
\hline \multirow{3}{*}{$\begin{array}{l}0 \\
\text { d } \\
\frac{1}{c} \\
\text { D } \\
\sum\end{array}$} & Agonist (ms) & $22,4 \pm 20,2$ & $-79,4 \pm 11,7$ & $-61,7 \pm 14,9$ & $-65,6 \pm 13,3$ \\
\hline & Antagonist (ms) & $126 \pm 17,8$ & $-25,2 \pm 11,1$ & $-1,9 \pm 17,8$ & $-9,2 \pm 17,6$ \\
\hline & Negativity (ms) & $-110 \pm 20,0$ & $189 \pm 26,8$ & $-\quad \pm$ & $\pm \quad-$ \\
\hline \multirow{3}{*}{ 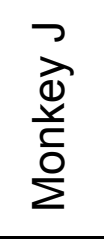 } & Agonist (ms) & $-3,57 \pm 13,9$ & $-93,7 \pm 18,3$ & $-111 \pm 16,7$ & $-93,4 \pm 15,3$ \\
\hline & Antagonist (ms) & $153 \pm 13,0$ & $-14,4 \pm 16,0$ & $25,8 \pm 14,5$ & $13,0 \pm 16,7$ \\
\hline & Negativity (ms) & $-93,9 \pm 11,1$ & $246 \pm 23,4$ & \pm & \pm \\
\hline \multirow{3}{*}{ 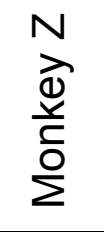 } & Agonist (ms) & $-42,9 \pm 15,8$ & $-101 \pm 14,5$ & $-112 \pm 14,8$ & $-117 \pm 12,1$ \\
\hline & Antagonist (ms) & $160 \pm 16,1$ & $36,7 \pm 17,1$ & $43,6 \pm 16,7$ & $50,1 \pm 18,1$ \\
\hline & Negativity (ms) & $-82,5 \pm 12,3$ & $213 \pm 21,9$ & \pm & \pm \\
\hline
\end{tabular}

649 Figure 6 Supplemental Table 1. Muscle activation and deactivation. Mean values $( \pm$ SD)

650 recorded for each monkey, and both groups of humans ( $\mathrm{n}=8$ in each group) are presented for 651 downward, upward, leftward and rightward movements. Timings were computed with respect 652 to movement onset. For each direction, agonist and antagonist muscle activation timings and 653 antigravity muscle deactivation (negativity) are provided. 


\begin{tabular}{|c|c|c|c|c|c|}
\hline & & \multicolumn{2}{|c|}{ Direction effect } & \multicolumn{2}{|c|}{ Post-hoc (Scheffe) } \\
\hline & & $\mathrm{F}$ & $\mathrm{P}$ & Up-Down & Right-Left \\
\hline \multirow{2}{*}{$\begin{array}{l}\text { Humans } \\
\text { ego-paralell }\end{array}$} & AG onset & 43,3 & $<1 \mathrm{E}-06$ & $<1 \mathrm{E}-06$ & 0,98 \\
\hline & ANT onset & 63,0 & $<1 \mathrm{E}-06$ & $<1 \mathrm{E}-06$ & 0,70 \\
\hline \multirow{2}{*}{$\begin{array}{c}\text { Humans } \\
\text { ego-perpendicular }\end{array}$} & AG onset & 25,2 & $<1 \mathrm{E}-06$ & $<8 E-06$ & 0,99 \\
\hline & ANT onset & 52,6 & $<1 \mathrm{E}-06$ & $<1 \mathrm{E}-06$ & 0,92 \\
\hline \multirow{2}{*}{ Monkey D } & AG onset & 83,4 & $<1 \mathrm{E}-06$ & $<1 \mathrm{E}-06$ & 0,90 \\
\hline & ANT onset & 115,3 & $<1 \mathrm{E}-06$ & $<1 \mathrm{E}-06$ & 0,52 \\
\hline \multirow{2}{*}{ Monkey J } & AG onset & 96,8 & $<1 \mathrm{E}-06$ & $<1 \mathrm{E}-06$ & 0,12 \\
\hline & ANT onset & 241,8 & $<1 \mathrm{E}-06$ & $<1 \mathrm{E}-06$ & 0,32 \\
\hline \multirow{2}{*}{ Monkey Z } & AG onset & 81,9 & $<1 \mathrm{E}-06$ & $<1 \mathrm{E}-06$ & 0,96 \\
\hline & ANT onset & 119,0 & $<1 \mathrm{E}-06$ & $<1 \mathrm{E}-06$ & 0,86 \\
\hline
\end{tabular}

655 Figure 6 Supplemental Table 2. Statistical comparisons on activation timings. F and P

656 values resulting from an ANOVA test on direction effect are presented for all parameters 657 presented in Fig.6 Suppl. Table 1. Results of Post-hoc analysis (Scheffe) on opposed 658 directions in the two planes (Up vs Down \& Right vs Left) are also reported. Similarly to 659 endpoint kinematics, humans $(\mathrm{H})$ and monkeys (D, J \& Z) exhibited directional asymmetries 660 in the earth-vertical plane but not in the earth-horizontal plane. 
Acceleration

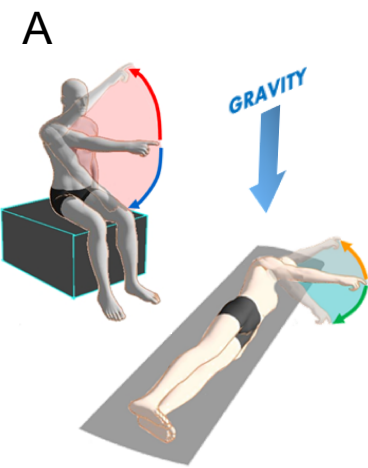

B

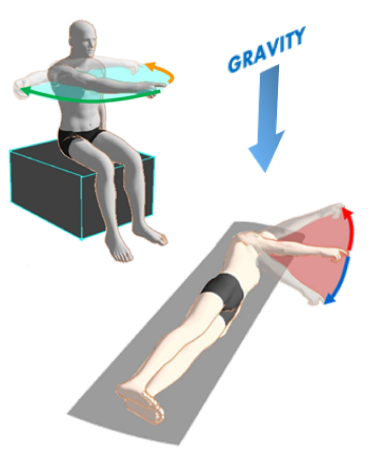

C

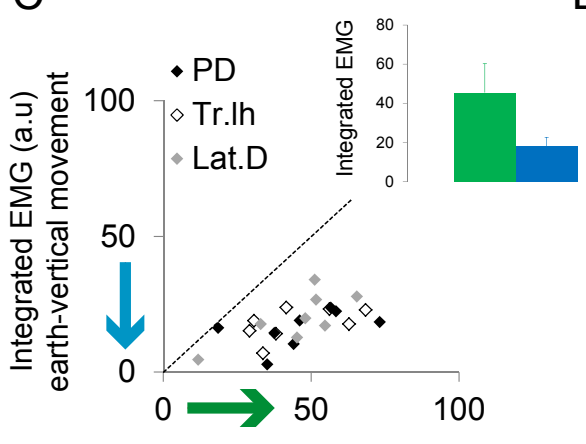

$\mathrm{D}$

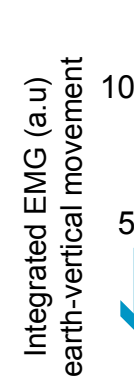

Deceleration

$E$

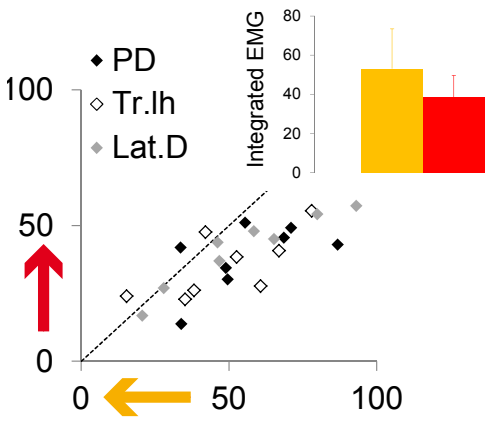

Integrated EMG (a.u) earth-horizontal movement

661

662

663

664

665

666

667

668

669

670

671

672

673

674

675

676

677

678

679

Figure 7. Saving effects on muscles pulling downwards. Activations of the agonist and antagonist muscles for both groups of humans ( 3 muscles and 8 participants per group) during earth-vertical and earth-horizontal movements. A. Experimental setup and movement directions in the ego-parallel group. B. Experimental setup and movement directions in the ego-perpendicular group. In both groups, the $90^{\circ}$ body tilt in roll allows comparing activations of the same muscles during reciprocal movements performed in the earth-vertical and the earth-horizontal planes. Coloured arrows indicate movement and gravity directions. C. Ego-parallel group. For each participant $(\mathrm{n}=8)$ and three agonist muscles (PD, Posterior Deltoid; Tr.lh, Triceps Long Head; Lat.D, Latissimus Dorsi), the mean normalised muscular activations $( \pm \mathrm{SD})$ integrated over the acceleration phase towards the feet are compared between body orientations. Downwards earth-vertical movements (vertical axis) are compared to rightwards earth-horizontal movements (horizontal axis). The bar-graph insert presents mean activations of all muscles and participants for the two body orientations. D. Egoperpendicular group. For each participant $(n=8)$ and three agonist muscles (MD, Middle Deltoid; Infra., Infraspinatus; BB.lh, long head of Biceps Brachii), the mean normalised muscular activations $( \pm \mathrm{SD})$ integrated over the acceleration phase towards the left side of the body are compared between body orientations. Downwards earth-vertical movements (vertical axis) are compared to leftwards earth-horizontal movements (horizontal axis). The 
680 bar-graph insert presents mean activations of all muscles and participants for the two body

681 orientations. D. Ego-parallel group. Mean normalised antagonist muscle activations ( \pm SD)

682 integrated over the deceleration phase towards the head are compared between body

683 orientations. Upwards earth-vertical movements (vertical axis) are compared to leftwards

684 earth-horizontal movements (horizontal axis). The bar-graph insert presents mean activations

685 of all muscles $(\mathrm{n}=3)$ and participants $(\mathrm{n}=8)$ for the two body orientations. E. Ego-

686 perpendicular group. Mean normalised antagonist muscle activations $( \pm$ SD) integrated over

687 the deceleration phase towards the right side of the body are compared between body

688 orientations. Upwards earth-vertical movements (vertical axis) are compared to rightwards

689 earth-horizontal movements (horizontal axis). The bar-graph insert presents mean activations

690 of all muscles $(n=3)$ and participants $(n=8)$ for the two body orientations. The use of gravity

691 force for downwards acceleration and upwards deceleration (as revealed by negatives phases

692 on antigravity muscles in previous figures) results in reduced muscle activations in the earth-

693 vertical compared to the earth-horizontal plane. 


\section{Acknowledgements}

695 This work was supported by the Institut National de la Santé et de la Recherche Médicale

696 (INSERM), by the Agence National de Recherche (ANR, project MOTION ANR-14-CE30-

697 0007-01), by the Centre National d'Etudes Spatiales (CNES) and by National Institute of

698 Health Grant R01-AT010459. We thank Daniel Moran and Thomas Pearce for their technical

699 support.

700

701 Competing interests: the authors declare no competing financial or non-financial

702 interests. 
703

704

705

706

707

708

709

710

711

712

713

714

715

716

717

718

719

720

721

722

723

724

725

726

727

728

729

730

731

\section{Bibliography}

Agostino, R., Hallett, M., and Sanes, J.N. (1992). Antagonist muscle inhibition before rapid voluntary movements of the human wrist. Electroencephalogr. Clin. Neurophysiol. Evoked Potentials.

Angelaki, D.E., McHenry, M.Q., Dickman, J.D., Newlands, S.D., and Hess, B.J. (1999). Computation of inertial motion: neural strategies to resolve ambiguous otolith information. J Neurosci 19, 316-327.

Angelaki, D.E., Shaikh, A.G., Green, A.M., and Dickman, J.D. (2004). Neurons compute internal models of the physical laws of motion. Nature 430, 560-564. Atkeson, C.G., and Hollerbach, J.M. (1985). Kinematic features of unrestrained vertical arm movements. J Neurosci 5, 2318-2330.

Baraduc, P., Thobois, S., Gan, J., Broussolle, E., and Desmurget, M. (2013). A Common Optimization Principle for Motor Execution in Healthy Subjects and Parkinsonian Patients. J. Neurosci.

Berardelli, A., Hallett, M., Rothwell, J.C., Agostino, R., Manfredi, M., Thompson, P.D., and Marsden, C.D. (1996). Single-joint rapid arm movements in normal subjects and in patients with motor disorders. Brain.

Berghänel, A., Schülke, O., and Ostner, J. (2015). Locomotor play drives motor skill acquisition at the expense of growth: A life history trade-off. Sci. Adv. Bernstein, N. (1967). The coordination and regulation of movements (Oxford). Berret, B., Darlot, C., Jean, F., Pozzo, T., Papaxanthis, C., and Gauthier, J.P. (2008). The inactivation principle: mathematical solutions minimizing the absolute work and biological implications for the planning of arm movements. PLoS Comput Biol 4, e1000194.

Bramble, D.M., and Lieberman, D.E. (2004). Endurance running and the evolution of Homo. Nature 432, 345-352.

Bringoux, L., Blouin, J., Coyle, T., Ruget, H., and Mouchnino, L. (2012). Effect of gravity-like torque on goal-directed arm movements in microgravity. J. Neurophysiol. 107, 2541-2548. 
732 Brown, J.H., Gillooly, J.F., Allen, A.P., Savage, V.M., and West, G.B. (2004).

733 Toward a metabolic theory of ecology. In Ecology, p.

734 Buneo, C.A., Soechting, J.F., and Flanders, M. (1994). Muscle activation

735 patterns for reaching: the representation of distance and time. J Neurophysiol 71, 736 1546-1558.

737 Burdet, E., Osu, R., Franklin, D.W., Milner, T.E., and Kawato, M. (2001). The

738 central nervous system stabilizes unstable dynamics by learning optimal

739 impedance. Nature 414, 446-449.

740 Carrier, D.R., Anders, C., and Schilling, N. (2011). The musculoskeletal system

741 of humans is not tuned to maximize the economy of locomotion. Proc Natl Acad

742 Sci U S A 108, 18631-18636.

743 Cavanagh, P.R., and Komi, P. V (1979). Electromechanical delay in human

744 skeletal muscle under concentric and eccentric contractions. Eur J Appl Physiol

745 Occup Physiol 42, 159-163.

746 Cheval, B., Tipura, E., Burra, N., Frossard, J., Chanal, J., Orsholits, D., Radel,

747 R., and Boisgontier, M.P. (2018a). Avoiding sedentary behaviors requires more

748 cortical resources than avoiding physical activity: An EEG study.

749 Neuropsychologia.

750 Cheval, B., Radel, R., Neva, J.L., Boyd, L.A., Swinnen, S.P., Sander, D., and

751 Boisgontier, M.P. (2018b). Behavioral and Neural Evidence of the Rewarding

752 Value of Exercise Behaviors: A Systematic Review. Sport. Med.

753 Chiovetto, E., Berret, B., and Pozzo, T. (2010). Tri-dimensional and triphasic

754 muscle organization of whole-body pointing movements. Neuroscience.

755 Cook, J.L., Blakemore, S.J., and Press, C. (2013). Atypical basic movement

756 kinematics in autism spectrum conditions. Brain 136, 2816-2824.

757 Cooke, J.D., and Brown, S.H. (1994). Movement-related phasic muscle

758 activation. III. The duration of phasic agonist activity initiating movement. Exp

759 Brain Res 99, 473-482.

760 Corcos, D.M., Gottlieb, G.L., and Agarwal, G.C. (1989). Organizing principles 
761 for single-joint movements. II. A speed-sensitive strategy. J Neurophysiol 62, $762 \quad 358-368$.

763 Crevecoeur, F., Thonnard, J.L., and Lefevre, P. (2009). Optimal integration of 764 gravity in trajectory planning of vertical pointing movements. J Neurophysiol $765102,786-796$.

766 d'Avella, A., Portone, A., Fernandez, L., and Lacquaniti, F. (2006). Control of 767 fast-reaching movements by muscle synergy combinations. J Neurosci 26, $7687791-7810$.

769 d'Avella, A., Fernandez, L., Portone, A., and Lacquaniti, F. (2008). Modulation 770 of phasic and tonic muscle synergies with reaching direction and speed. J 771 Neurophysiol 100, 1433-1454.

772 Dakin, C.J., and Rosenberg, A. (2018). Gravity estimation and verticality 773 perception. Handb Clin Neurol 159, 43-59.

774 David, F.J., Robichaud, J.A., Vaillancourt, D.E., Poon, C., Kohrt, W.M., 775 Comella, C.L., and Corcos, D.M. (2016). Progressive resistance exercise 776 restores some properties of the triphasic EMG pattern and improves 777 bradykinesia: the PRET-PD randomized clinical trial. J. Neurophysiol. 778 DeLong, J.P., Okie, J.G., Moses, M.E., Sibly, R.M., and Brown, J.H. (2010).

779 Shifts in metabolic scaling, production, and efficiency across major evolutionary 780 transitions of life. Proc. Natl. Acad. Sci.

781 Edey, R., Cook, J., Brewer, R., Bird, G., and Press, C. (2019). Adults with 782 autism spectrum disorder are sensitive to the kinematic features defining natural 783 human motion. Autism Res.

784 Elmore, L.C., Cassidy, R.M., Rosenberg, A., Deangelis, G.C., and Angelaki, 785 D.E. (2014). Perception of planar surface orientation relative to earth vertical. 786 Progr. No. 822.02. 2014 Neurosci. Meet. Planner. Washington, DC Soc. 787 Neurosci. 2014. Online.

788 Farshchiansadegh, A., Melendez-Calderon, A., Ranganathan, R., Murphey, 789 T.D., and Mussa-Ivaldi, F.A. (2016). Sensory Agreement Guides Kinetic Energy 
Optimization of Arm Movements during Object Manipulation. PLoS Comput Biol 12, e1004861.

Flanders, M., and Herrmann, U. (1992). Two components of muscle activation: scaling with the speed of arm movement. J Neurophysiol 67, 931-943.

Flanders, M., Pellegrini, J.J., and Soechting, J.F. (1994). Spatial/temporal characteristics of a motor pattern for reaching. J Neurophysiol 71, 811-813. Flanders, M., Pellegrini, J.J., and Geisler, S.D. (1996). Basic features of phasic activation for reaching in vertical planes. Exp Brain Res 110, 67-79. Forgaard, C.J., Maslovat, D., Carlsen, A.N., Chua, R., and Franks, I.M. (2013). Startle reveals independent preparation and initiation of triphasic EMG burst components in targeted ballistic movements. J. Neurophysiol.

Franklin, D.W., and Wolpert, D.M. (2011). Computational mechanisms of sensorimotor control. Neuron.

Gaveau, J., and Papaxanthis, C. (2011). The temporal structure of vertical arm movements. PLoS One 6.

Gaveau, J., Paizis, C., Berret, B., Pozzo, T., and Papaxanthis, C. (2011).

Sensorimotor adaptation of point-to-point arm movements after spaceflight:The role of internal representation of gravity force in trajectory planning. J.

Neurophysiol. 106.

Gaveau, J., Berret, B., Demougeot, L., Fadiga, L., Pozzo, T., and Papaxanthis, C. (2014). Energy-related optimal control accounts for gravitational load: Comparing shoulder, elbow, and wrist rotations. J. Neurophysiol. 111.

Gaveau, J., Berret, B., Angelaki, D.E., and Papaxanthis, C. (2016). Directiondependent arm kinematics reveal optimal integration of gravity cues. Elife 5 . Gentili, R., Cahouet, V., and Papaxanthis, C. (2007). Motor planning of arm movements is direction-dependent in the gravity field. Neuroscience 145,20 32.

Guigon, E., Baraduc, P., and Desmurget, M. (2007). Computational motor control: redundancy and invariance. J. Neurophysiol. 97, 331-347. 
819 Guigon, E., Baraduc, P., and Desmurget, M. (2008). Optimality, stochasticity,

820 and variability in motor behavior. J. Comput. Neurosci.

821 Hagen, D.A., and Valero-Cuevas, F.J. (2017). Similar movements are associated

822 with drastically different muscle contraction velocities. J. Biomech.

823 Hollerbach, M.J., and Flash, T. (1982). Dynamic interactions between limb

824 segments during planar arm movement. Biol Cybern 44, 67-77.

825 Hondzinski, J.M., Soebbing, C.M., French, A.E., and Winges, S.A. (2016).

826 Different damping responses explain vertical endpoint error differences between 827 visual conditions. Exp. Brain Res.

828 Huang, H.J., Kram, R., and Ahmed, A.A. (2012). Reduction of metabolic cost

829 during motor learning of arm reaching dynamics. J Neurosci 32, 2182-2190.

830 Huang, Y.Z., Chang, Y.S., Hsu, M.J., Wong, A.M.K., and Chang, Y.J. (2015).

831 Restoration of central programmed movement pattern by temporal electrical

832 stimulation-assisted training in patients with spinal cerebellar atrophy. Neural

833 Plast.

834 Indovina, I., Maffei, V., Bosco, G., Zago, M., Macaluso, E., and Lacquaniti, F.

835 (2005). Representation of visual gravitational motion in the human vestibular 836 cortex. Science (80-. ). 308, 416-419.

837 Inzlicht, M., Shenhav, A., and Olivola, C.Y. (2018). The Effort Paradox: Effort 838 Is Both Costly and Valued. Trends Cogn. Sci.

839 Irlbacher, K., Voss, M., Meyer, B.U., and Rothwell, J.C. (2006). Influence of 840 ipsilateral transcranial magnetic stimulation on the triphasic EMG pattern 841 accompanying fast ballistic movements in humans. J Physiol 574, 917-928.

842 Izawa, J., Rane, T., Donchin, O., and Shadmehr, R. (2008). Motor adaptation as 843 a process of reoptimization. J Neurosci 28, 2883-2891.

844 Jorges, B., and Lopez-Moliner, J. (2017). Gravity as a Strong Prior: Implications 845 for Perception and Action. Front Hum Neurosci 11, 203.

846 Kadmon Harpaz, N., Flash, T., and Dinstein, I. (2014). Scale-invariant

847 movement encoding in the human motor system. Neuron 81, 452-462. 
848 Kelso, J.A.S., Southard, D.L., and Goodman, D. (1979). On the nature of human

849 interlimb coordination. Science (80-. ).

850 Klein, J., Whitsell, B., Artemiadis, P.K., and Buneo, C.A. (2018). Perception of

851 Arm Position in Three-Dimensional Space. Front Hum Neurosci 12, 331.

852 Krabben, T., Prange, G.B., Molier, B.I., Stienen, A.H., Jannink, M.J., Buurke, 853 J.H., and Rietman, J.S. (2012). Influence of gravity compensation training on 854 synergistic movement patterns of the upper extremity after stroke, a pilot study. 855 J Neuroeng Rehabil 9, 44.

856 Kurtzer, I., Herter, T.M., and Scott, S.H. (2006). Nonuniform distribution of 857 reach-related and torque-related activity in upper arm muscles and neurons of 858 primary motor cortex. J Neurophysiol 96, 3220-3230.

859 Kurzban, R., Duckworth, A., Kable, J.W., and Myers, J. (2013). An opportunity 860 cost model of subjective effort and task performance. Behav Brain Sci 36, 661861679.

862 Lacquaniti, F., Bosco, G., Indovina, I., La Scaleia, B., Maffei, V., Moscatelli, 863 A., and Zago, M. (2013). Visual gravitational motion and the vestibular system 864 in humans. Front Integr Neurosci 7, 101.

865 Laurens, J., Meng, H., and Angelaki, D.E. (2013a). Neural representation of 866 orientation relative to gravity in the macaque cerebellum. Neuron $80,1508-$ 8671518.

868 Laurens, J., Meng, H., and Angelaki, D.E. (2013b). Computation of linear 869 acceleration through an internal model in the macaque cerebellum. Nat Neurosci $87016,1701-1708$.

871 Laurens, J., Kim, B., Dickman, J.D., and Angelaki, D.E. (2016). Gravity 872 orientation tuning in macaque anterior thalamus. Nat Neurosci 19, 1566-1568.

873 Lee, H.H., Emerson, J.A., and Williams, D.M. (2016). The exercise-affect874 adherence pathway: An evolutionary perspective. Front. Psychol.

875 Lieberman, D.E. (2015). Is Exercise Really Medicine? An Evolutionary 876 Perspective. Curr. Sports Med. Rep. 
877 Manckoundia, P., Mourey, F., Pfitzenmeyer, P., and Papaxanthis, C. (2006).

878 Comparison of motor strategies in sit-to-stand and back-to-sit motions between

879 healthy and Alzheimer's disease elderly subjects. Neuroscience 137, 385-392.

880 Mazzoni, P., Hristova, A., and Krakauer, J.W. (2007). Why don't we move

881 faster? Parkinson's disease, movement vigor, and implicit motivation. J

882 Neurosci 27, 7105-7116.

883 McIntyre, J., Zago, M., Berthoz, A., and Lacquaniti, F. (2001). Does the brain 884 model Newton's laws? Nat Neurosci 4, 693-694.

885 Miller, W.L., Maffei, V., Bosco, G., Iosa, M., Zago, M., Macaluso, E., and 886 Lacquaniti, F. (2008). Vestibular nuclei and cerebellum put visual gravitational 887 motion in context. J. Neurophysiol. 99, 1969-1982.

888 Moran, D.W., and Schwartz, A.B. (1999). Motor cortical representation of speed 889 and direction during reaching. J Neurophysiol 82, 2676-2692.

890 Morel, P., Ulbrich, P., and Gail, A. (2017). What makes a reach movement 891 effortful? Physical effort discounting supports common minimization principles 892 in decision making and motor control. PLoS Biol.

893 Munch, S.B., and Salinas, S. (2009). Latitudinal variation in lifespan within 894 species is explained by the metabolic theory of ecology. Proc. Natl. Acad. Sci.

895 Olesh, E. V., Pollard, B.S., and Gritsenko, V. (2017). Gravitational and 896 Dynamic Components of Muscle Torque Underlie Tonic and Phasic Muscle 897 Activity during Goal-Directed Reaching. Front. Hum. Neurosci.

898 Pageaux, B. (2016). Perception of effort in Exercise Science: Definition, 899 measurement and perspectives. Eur. J. Sport Sci.

900 Pageaux, B., and Gaveau, J. (2016). Studies using pharmacological blockade of 901 muscle afferents provide new insights into the neurophysiology of perceived 902 exertion. J. Physiol. 594.

903 Papaxanthis, C., Pozzo, T., Popov, K.E., and McIntyre, J. (1998a). Hand 904 trajectories of vertical arm movements in one-G and zero-G environments. 905 Evidence for a central representation of gravitational force. Exp Brain Res 120, 
496-502.

907 Papaxanthis, C., Pozzo, T., Vinter, A., and Grishin, A. (1998b). The

908 representation of gravitational force during drawing movements of the arm. Exp 909 Brain Res 120, 233-242.

910 Papaxanthis, C., Dubost, V., and Pozzo, T. (2003). Similar planning strategies

911 for whole-body and arm movements performed in the sagittal plane.

912 Neuroscience 117, 779-783.

913 Van Pelt, S., Van Gisbergen, J.A., and Medendorp, W.P. (2005). Visuospatial 914 memory computations during whole-body rotations in roll. J Neurophysiol 94 , $915 \quad 1432-1442$.

916 Prange, G.B., Jannink, M.J., Stienen, A.H., van der Kooij, H., Ijzerman, M.J., 917 and Hermens, H.J. (2009a). Influence of gravity compensation on muscle 918 activation patterns during different temporal phases of arm movements of stroke 919 patients. Neurorehabil Neural Repair 23, 478-485.

920 Prange, G.B., Kallenberg, L.A., Jannink, M.J., Stienen, A.H., van der Kooij, H., 921 Ijzerman, M.J., and Hermens, H.J. (2009b). Influence of gravity compensation 922 on muscle activity during reach and retrieval in healthy elderly. J Electromyogr 923 Kinesiol 19, e40-9.

924 Prange, G.B., Krabben, T., Renzenbrink, G.J., Ijzerman, M.J., Hermens, H.J., 925 and Jannink, M.J. (2012). Changes in muscle activation after reach training with 926 gravity compensation in chronic stroke patients. Int J Rehabil Res 35, 234-242. 927 Raj, S., Dounskaia, N., Clark, W.W., and Sethi, A. (2019). Effect of Stroke on 928 Joint Control during Reach-to-Grasp: A Preliminary Study. J. Mot. Behav. 929 Rosenberg, A., and Angelaki, D.E. (2014). Gravity influences the visual 930 representation of object tilt in parietal cortex. J Neurosci 34, 14170-14180.

931 Rousseau, C., Fautrelle, L., Papaxanthis, C., Fadiga, L., Pozzo, T., and White, 932 O. (2016). Direction-dependent activation of the insular cortex during vertical 933 and horizontal hand movements. Neuroscience 325, 10-19.

934 Russo, M., D’Andola, M., Portone, A., Lacquaniti, F., and d'Avella, A. (2014). 
935 Dimensionality of joint torques and muscle patterns for reaching. Front Comput

936 Neurosci 8, 24.

937 Saradjian, A.H., Paleressompoulle, D., Louber, D., Coyle, T., Blouin, J., and

938 Mouchnino, L. (2014). Do gravity-related sensory information enable the

939 enhancement of cortical proprioceptive inputs when planning a step in

940 microgravity? PLoS One.

941 La Scaleia, B., Lacquaniti, F., and Zago, M. (2019). Body orientation

942 contributes to modelling the effects of gravity for target interception in humans.

943 J Physiol 597, 2021-2043.

944 Sciutti, A., Demougeot, L., Berret, B., Toma, S., Sandini, G., Papaxanthis, C.,

945 and Pozzo, T. (2012). Visual gravity influences arm movement planning. J.

946 Neurophysiol. 107, 3433-3445.

947 Le Seac'h, A.B., and McIntyre, J. (2007). Multimodal reference frame for the

948 planning of vertical arms movements. Neurosci Lett 423, 211-215.

949 Selinger, J.C., O’Connor, S.M., Wong, J.D., and Donelan, J.M. (2015). Humans

950 Can Continuously Optimize Energetic Cost during Walking. Curr Biol 25, $9512452-2456$.

952 Senot, P., Zago, M., Lacquaniti, F., and McIntyre, J. (2005). Anticipating the

953 effects of gravity when intercepting moving objects: differentiating up and down

954 based on nonvisual cues. J Neurophysiol 94, 4471-4480.

955 Shadmehr, R., and Mussa-Ivaldi, F.A. (1994). Adaptive representation of

956 dynamics during learning of a motor task. J Neurosci 14, 3208-3224.

957 Shadmehr, R., and Wise, S.P. (2005). The computational neurobiology of 958 reaching and pointing (London: The MIT Press).

959 Shadmehr, R., Smith, M.A., and Krakauer, J.W. (2010). Error Correction,

960 Sensory Prediction, and Adaptation in Motor Control. Annu. Rev. Neurosci.

961 Shadmehr, R., Huang, H.J., and Ahmed, A.A. (2016). A Representation of

962 Effort in Decision-Making and Motor Control. Curr Biol 26, 1929-1934.

963 Snaterse, M., Ton, R., Kuo, A.D., and Donelan, J.M. (2011). Distinct fast and 
964 slow processes contribute to the selection of preferred step frequency during 965 human walking. J Appl Physiol 110, 1682-1690.

966 Strotz, L.C., Saupe, E.E., Kimmig, J., and Lieberman, B.S. (2018). Metabolic 967 rates, climate and macroevolution: a case study using Neogene molluscs.

968 Proceedings. Biol. Sci.

969 Tajadura-Jimenez, A., Deroy, O., Marquardt, T., Bianchi-Berthouze, N., Asai, 970 T., Kimura, T., and Kitagawa, N. (2018). Audio-tactile cues from an object's 971 fall change estimates of one's body height. PLoS One 13, e0199354.

972 Todorov, E. (2004). Optimality principles in sensorimotor control. Nat Neurosci 973 7, 907-915.

974 Voorhies, W. Van, and Ward, S. (1999). Genetic and environmental conditions 975 that increase longevity in Caenorhabditis elegans decrease metabolic rate. Proc. 976 Natl. Acad. Sci. U. S. A.

977 De Vrijer, M., Medendorp, W.P., and Van Gisbergen, J.A. (2008). Shared 978 computational mechanism for tilt compensation accounts for biased verticality 979 percepts in motion and pattern vision. J Neurophysiol 99, 915-930.

980 Walton, M.E., Kennerley, S.W., Bannerman, D.M., Phillips, P.E., and 981 Rushworth, M.F. (2006). Weighing up the benefits of work: behavioral and 982 neural analyses of effort-related decision making. Neural Netw. 19, 1302-1314. 983 Wang, W., and Dounskaia, N. (2012). Load emphasizes muscle effort 984 minimization during selection of arm movement direction. J. Neuroeng. Rehabil. 985 Wang, W., Chan, S.S., Heldman, D.A., and Moran, D.W. (2010). Motor cortical 986 representation of hand translation and rotation during reaching. J Neurosci 30, 987 958-962.

988 Wolpert, D.M., and Ghahramani, Z. (2000). Computational principles of 989 movement neuroscience. Nat. Neurosci.

990 Yamamoto, S., and Kushiro, K. (2014). Direction-dependent differences in 991 temporal kinematics for vertical prehension movements. Exp Brain Res 232, 992 703-711. 